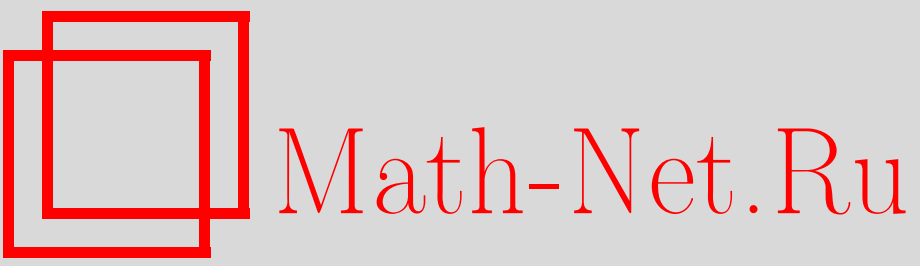

А. А. Гущин, Двойственная характеризация цены в задаче максимизации робастной полезности, Теория вероятн. и ее примен., 2010, том 55, выпуск 4, 680704

DOI: https://doi.org/10.4213/tvp4278

Использование Общероссийского математического портала Math-Net.Ru подразумевает, что вы прочитали и согласны с пользовательским соглашением http://www.mathnet.ru/rus/agreement

Параметры загрузки:

IP: 44.207 .124 .84

26 апреля 2023 г., 15:37:37 


\title{
ДВОЙСТВЕННАЯ ХАРАКТЕРИЗАЦИЯ ЦЕНЫ В ЗАДАЧЕ МАКСИМИЗАЦИИ РОБАСТНОЙ ПОЛЕЗНОСТИ ${ }^{1)}$
}

\begin{abstract}
Рассматривается задача максимизации робастной полезности от терминального капитала в случае, когда рынок характеризуется только множеством терминальных капиталов, отвечающих всем допустимым стратегиям инвестора. Основное внимание уделено случаю, когда функция полезности конечна на полупрямой. Доказана минимаксная теорема, сводящая робастную постановку к стандартной, приведена двойственная характеризация цены в исходной задаче и доказаны те свойства решений и функций цены для исходной и двойственной задач, которые не требуют дополнительных предположений типа условий на асимптотическую эластичность функции полезности.
\end{abstract}

Ключевые слова и фразы: двойственная задача, $f$-дивергенция, максимизация полезности, робастная полезность.

1. Введение. В этой статье под задачей максимизации робастной полезности (со штрафной функцией) мы понимаем задачу максимизации функционала

$$
\xi \rightsquigarrow \inf _{\mathbf{Q} \in \mathscr{Q}}\left[\mathbf{E}_{\mathbf{Q}} U(\xi)+\gamma(\mathbf{Q})\right], \quad \xi \in \mathscr{A},
$$

по некоторому выпуклому множеству $\mathscr{A}$ случайных величин, заданных на вероятностном пространстве $(\Omega, \mathscr{F}, \mathbf{P})$, где функция полезности $U$ определена на $\mathbf{R}$ и принимает значения в $\mathbf{R} \cup\{-\infty\}$, вогнута и монотонно не убывает, $\mathscr{Q}$ - некоторое выпуклое множество «субъективных» вероятностных мер на $(\Omega, \mathscr{F})$, штрафная функция $\gamma$ выпукла; более точные предположения сформулированы в разд. 2. Здесь и далее математическое ожидание $\mathbf{E}_{\mathbf{Q}}$ считается равным $-\infty$, если оно не определено, т.е. $\mathbf{E}_{\mathbf{Q}} \eta=\lim _{n \rightarrow+\infty} \mathbf{E}_{\mathbf{Q}}(\eta \wedge n)$. Если $\gamma(\mathbf{Q}) \equiv 0$ на $\mathscr{Q}$, говорят также о максимизации когерентной робастной полезности (термин «когерентная» происходит из терминологии, принятой в теории выпуклых мер риска). Под стандартной задачей максимизации полезности будем понимать случай, когда $\mathscr{Q}=\{\mathbf{P}\}$ или множество $\mathscr{Q}$ состоит из одной меры.

\footnotetext{
*Математический институт им. В.А.Стеклова РАН, ул. Губкина, 8, 119991 Москва, Россия; e-mail: gushchin@mi.ras.ru

1) Работа выполнена при поддержке РФФИ (гранты № 05-01-00944, 08-01-00740 и 08-01-91205).
} 
Элементы множества $\mathscr{A}$ можно интерпретировать как капитал инвестора в заключительный момент операций на финансовом рынке (терминальный капитал), тогда $\mathscr{A}$ интерпретируется как множество терминальных капиталов инвестора, отвечающих его всевозможным (допустимым) стратегиям, а о задаче максимизации функционала (1.1) говорят как о задаче максимизации ожидаемой полезности от терминального капитала. Другие интерпретации задачи также возможны, хотя наши предположения ориентированы, в первую очередь, именно на указанную ситуацию. При обзоре литературы ниже мы упоминаем также статьи, в которых рассматриваются более общие задачи, включающие минимизацию полезности как от терминального капитала, так и, скажем, от процесса потребления.

Функционал (1.1) отражает предпочтения инвестора, не склонного к риску, происходящему как от случайности (за что отвечает вогнутость $U)$, так и от неопределенности вероятностной модели, действующей на рынке (взятие нижней грани по $\mathscr{Q}$ означает, что инвестор оценивает стратегию по наихудшему из всех субъективных вероятностных сценариев $\mathbf{Q} \in \mathscr{Q}$ ). Аксиоматическое описание предпочтений инвестора, приводящих к численному представлению этих предпочтений в виде функционала типа (1.1), рассматривалось, в частности, в работах [43] (стандартная задача), [12] (когерентная робастная полезность), [24] (робастная полезность со штрафной функцией); см. также недавнюю работу [10].

Настоящая статья является первой из двух статей, в которых основное внимание уделяется задаче, двойственной к задаче максимизации робастной полезности от терминального капитала. Цель этих статей доказать ряд ключевых утверждений теории при как можно более слабых предположениях. В данной статье мы доказываем минимаксную теорему, позволяющую в определенном смысле свести робастную постановку к стандартной, приводим двойственную характеризацию цены в исходной задаче и доказываем те свойства решений и функций цены для исходной и двойственной задач, которые не требуют дополнительных предположений типа условий на асимптотическую эластичность функции полезности. В частности, условия существования решения в исходной задаче не изучаются. Модель финансового рынка представлена в самой общей форме, а именно, основным объектом является множество $\mathscr{A}$ терминальных капиталов инвестора, отвечающее всем его возможным стратегиям. Соответственно, в терминах этого множества (без дальнейшей структуризации) даются все ответы. Динамические модели рынка будут рассмотрены во второй статье, где, в частности, будут указаны условия, при которых двойственную задачу можно поставить в терминах супермартингальных плотностей или мер.

Для марковских моделей финансового рынка задача максимизации 
полезности может быть решена методами динамического программирования (см., например, классические работы Р. Мертона [25], [26] и П. Самуэльсона [32]). Альтернативой являются двойственные методы выпуклого анализа, которые не требуют почти никаких предположений о структуре модели. Они предлагают решить сначала вспомогательную (двойственную) задачу, что позволяет охарактеризовать решение исходной задачи и найти ее цену. Оборотной стороной этих методов является то обстоятельство, что результаты о решении исходной задачи носят характер утверждений типа существования и единственности и не позволяют, вообще говоря, найти конкретное решение (что, впрочем, реально только при специальном выборе модели и других атрибутов задачи). Отметим, что для робастной задачи двойственный подход имеет дополнительное преимущество: исходная задача есть задача минимаксного типа, т.е. задача на поиск седловой точки, в то время как двойственная задача есть (вообще говоря, более простая) задача минимизации. В некоторых из упомянутых ниже работ опять же для марковских моделей рынка уже двойственная задача решалась методами динамического программирования, что позволило решить и исходную задачу.

Применение двойственных методов в задачах стохастического управления инициировано в работе Ж.-М. Бисмута [3], а в задаче максимизации полезности - в работе С. Плиски [29]. Наиболее значимый вклад в развитие двойственных методов был внесен в работе Д.Крамкова и В. Шахермайера [22], где можно найти ссылки на предшествующую литературу. В частности, в этой статье впервые было отмечено и подтверждено контрпримерами, что в двойственной задаче недостаточно ограничиться вероятностными мерами. Также ими были разграничены на две группы ключевые утверждения теории: в одну группу вошли утверждения, справедливые при общих предположениях, во вторую утверждения, справедливые при дополнительных предположениях, например, при условии на асимптотическую эластичность функции полезности. Как отмечалось выше, мы в данной работе ограничиваемся только утверждениями из первой группы.

Из большого числа последующих публикаций по стандартной задаче максимизации полезности отметим работы [1], [2], [4], [8], [9], [13], [21], [23], [28], [33], [34], [46], [48], наиболее близко примыкающие к методам и результатам нашего исследования; даже относительно полный перечень публикаций привести в журнальной статье невозможно. Задачи максимизации когерентной робастной полезности изучались, например, в [6], [11], [14], [16], [27], [30], [35], [36], [39], [41], [42] и обзорной статье [37]. Наконец, задача максимизации робастной полезности со штрафной функцией рассматривалась в работах $[17],[38],[44]$ и др., см. также обзорную статью [40].

Задача максимизация полезности существенно различна в следую- 
щих двух случаях: 1) функция полезности $U$ конечна на полупрямой $(a,+\infty)$ и равна $-\infty$ на $(-\infty, a) ; 2)$ функция полезности $U$ конечна всюду на $\mathbf{R}$. В основной части статьи мы имеем дело с первым случаем, без ограничения общности полагая, как обычно, что $a=0$. Второй случай несколько проще в предположении, что каждый элемент $\mathscr{A}$ ограничен снизу (см. п. 2.5). Отказ от этого предположения приводит к существенно более сложной задаче. В этом случае результаты, аналогичные нашим и по формулировкам, и по схеме доказательств, недавно были получены И. С. Морозовым [27].

Остановимся на наиболее характерных особенностях настоящего исследования.

Мы рассматриваем общую модель рынка, характеризуемую четверкой $(\Omega, \mathscr{F}, \mathbf{P}, \mathscr{A})$, в данной статье и общую динамическую модель рынка (не требующую введения процессов цен базовых активов, а характеризуемую множеством процессов капитала инвестора, отвечающих его всевозможным допустимым стратегиям) в последующей статье, стараясь работать при как можно более общих предположениях. Это позволяет получать, в качестве следствий общих результатов, результаты для моделей рынка с дискретным и непрерывным временем, конечным и бесконечным временным горизонтом, с конечным и бесконечным числом базовых активов, с ограничениями на портфель и без таковых и т.д.

От функции полезности $U$ требуются только те свойства, которые будут необходимы для доказательства данного факта. Так, для доказательства минимаксной теоремы и большинства результатов о двойственности требуются только предположения, которые уже были упомянуты выше, а такие стандартные предположения, как дифференцируемость, строгая вогнутость и условия Инада, не требуются. А выполнение, скажем, условия Инада в нуле для $U$ требуется тогда, когда это необходимо, например, при доказательстве условия Инада в нуле для функции ожидаемой полезности $u$. Таким образом, даже для стандартной задачи максимизации полезности наша основная теорема 2.2 является обобщением своей предшественницы, теоремы 3.1 в [22]. Представляется, что наш подход делает более понятным взаимосвязь условий и их следствий. Например, стандартные утверждения о поведении функции $v$ на бесконечности и функции $u$ в нуле требуют разных условий, см. пункты (vii) и (viii) теоремы 2.2 .

Мы не налагаем априори никаких условий типа отсутствия арбитража. Конечно, частью фольклора является утверждение о том, что арбитраж делает задачу максимизации полезности тривиальной, см. обсуждение этого вопроса в статье [20]. Условия «тривиальности» задачи максимизации полезности в рассматриваемой нами общей модели сформулированы в п. 2.3.

Полученная нами характеризация цены в исходной задаче через 
функцию цены в двойственной задаче является полной. Действительно, обычным предположением (в стандартной задаче) является конечность функции ожидаемой полезности $u$ в одной точке (и, стало быть, всюду), тогда $u$ выражается через функцию цены $v$ двойственной задачи. Естественно поставить вопрос: а можем ли мы, зная $v$, определить, является ли $u$ всюду конечной или всюду бесконечной? В робастном случае условия, при которых $u$ выражается через $v$, носят еще более сложный характер. Мы доказываем, что (только при основных предположениях) соотношения двойственности между $u$ и $v$ всегда имеют место.

В доказательствах мы чаще используем выпуклый анализ в противовес вероятностным методам (что не является самоцелью, а, на наш взгляд, упрощает доказательства). В частности, активно используются конечно-аддитивные функции множества (что не является редкостью), понятие $f$-дивергенции (что тоже встречалось) и новый подход к ее определению, предложенный в [15] и позволяющий распространить это определение на конечно-аддитивные функции множества, что является новым (см. также дальнейшее развитие этой идеи в [27]). Следует подчеркнуть, что в основные результаты конечно-аддитивные функции множества не входят.

Наконец, еще одна характерная особенность наших доказательств состоит в том, что, по существу, минимаксная теорема и двойственная характеризация доказываются одновременно. Схема доказательств, скажем, в работах [41] и [38] такова, что доказывается минимаксная теорема, сводящая робастную постановку к стандартной; двойственная же характеризация для стандартной задачи была получена в [22] также с использованием минимаксной теоремы.

Всюду в работе предполагается заданным вероятностное пространство $(\Omega, \mathscr{F}, \mathbf{P})$. Через $L^{0}=L^{0}(\mathscr{F}, \mathbf{P})$ обозначается пространство классов эквивалентности (относительно равенства Р-п.н.) $\mathscr{F}$-измеримых случайных величин с действительными значениями, наделенное топологией сходимости по вероятности. Подчеркнем, что предел сходящейся в $L^{0}$ последовательности не может принимать бесконечных значений. Неравенства типа $\xi \leqslant f(\eta)$ для элементов $\xi, \eta \in L^{0}$ понимаются как неравенства $\mathbf{P}$-п.н. Банаховы пространства $L^{1}=L^{1}(\mathscr{F}, \mathbf{P})$ и $L^{\infty}=L^{\infty}(\mathscr{F}, \mathbf{P})$ понимаются в обычным смысле. Обозначим $\mathfrak{b} \mathfrak{a}=\mathfrak{b} \mathfrak{a}(\mathscr{F}, \mathbf{P})$ пространство таких ограниченных конечно-аддитивных функций множества $\mu: \mathscr{F} \rightarrow \mathbf{R}$, что

$$
A \in \mathscr{F}, \mathbf{P}(A)=0 \quad \Rightarrow \quad \mu(A)=0,
$$

с нормой полной вариации. Хорошо известно, что $\mathfrak{b a}$ является сопряженным к пространству $L^{\infty}$, причем двойственность задается соотношением

$$
\langle\xi, \mu\rangle:=\mu(\xi):=\int_{\Omega} \xi d \mu, \quad \mu \in \mathfrak{b a}, \quad \xi \in L^{\infty} .
$$


Подпространство $\mathfrak{b a}$, состоящее из счетно-аддитивных мер, обозначается $\mathfrak{c a}$. Для $\mu \in \mathfrak{b a}$ существует единственное разложение $\mu=\mu^{\mathrm{r}}+\mu^{\mathrm{s}}$ на счетно-аддитивную меру $\mu^{\mathrm{r}} \in \mathfrak{c} \mathfrak{a}$ и чисто конечно-аддитивную функ-

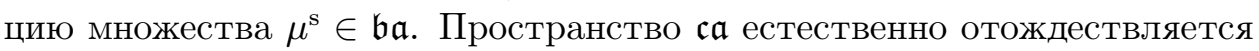
с $L^{1}$ посредством соотношения $L^{1} \ni \xi \rightsquigarrow \xi \cdot \mathbf{P} \in \mathfrak{c a}$, где $\xi \cdot \mathbf{P}$ есть мера с плотностью $\xi$ относительно $\mathbf{P}$.

Нижний индекс + у введенных функциональных пространств указывает на конус неотрицательных элементов (элементов с неотрицательными значениями).

Множество всех вероятностных мер на $(\Omega, \mathscr{F})$, абсолютно непрерывных относительно $\mathbf{P}$, обозначается $\mathfrak{P}$.

Упоминавшееся выше определение $f$-дивергенции $\mathscr{J}_{f}(\mu, \nu)$ для $\mu, \nu \in \mathfrak{b} \mathfrak{a}$ и ее свойства для удобства читателя приведены в приложении В (п. 4.2). В приложении А (п. 4.1) формулируются некоторые результаты из выпуклого анализа, используемые в статье. Здесь же мы поясним только те обозначения и определения, которые применяются при формулировке основных результатов. Для функции $f: X \rightarrow \mathbf{R} \cup\{+\infty\}$ эффективное множество $\operatorname{dom} f$ определяется как

$$
\operatorname{dom} f:=\{x \in X: f(x)<+\infty\} .
$$

Использование символа min вместо inf означает, что нижняя грань достигается. Индикаторные функции $\mathbf{1}_{A}$ и $\delta_{A}$ множества $A \subseteq X$ определяются следующим образом:

$$
\mathbf{1}_{A}(x):=\left\{\begin{array}{ll}
1, & \text { если } x \in X, \\
0, & \text { если } x \notin X,
\end{array} \quad \delta_{A}(x):= \begin{cases}0, & \text { если } x \in X, \\
+\infty, & \text { если } x \notin X .\end{cases}\right.
$$

\section{2. Основные результаты.}

2.1. Основные предположения. В этом пункте формулируются предположения о функции полезности, штрафной функции и множестве терминальных капиталов, при которых доказаны основные результаты работы, и вводятся необходимые обозначения.

П р е д п о ло ж е н и е 2.1. Функция полезности $U: \mathbf{R} \rightarrow \mathbf{R} \cup$ $\{-\infty\}-$ монотонно неубьвающая вогнутая функиия, $U(x)=-\infty$ nри $x<0$ u $U(x) \in \mathbf{R}$ при $x>0$.

Ясно, что функция $U$ непрерывна на $(0,+\infty)$. Непрерывность справа функции $U$ в точке 0 (что эквивалентно полунепрерывности сверху функции $U)$ не предполагается. Положим

$$
V(y)=\sup _{x>0}[U(x)-x y], \quad y \in \mathbf{R} .
$$


Иначе говоря, $V$ есть преобразование Фенхеля функции $-U(-\cdot)$. Поэтому $V: \mathbf{R} \rightarrow \mathbf{R} \cup\{+\infty\}$ - собственная полунепрерывная снизу выпуклая функция, а по теореме Фенхеля-Моро

$$
\inf _{y \in \mathbf{R}}[V(y)+x y]=\widetilde{U}(x):= \begin{cases}U(x), & \text { если } x \neq 0 ; \\ \lim _{x \downarrow 0} U(x), & \text { если } x=0 .\end{cases}
$$

Далее, в силу предположения $2.1 \operatorname{dom} V \subseteq \mathbf{R}_{+}, V$ монотонно не возрастает и

$$
\lim _{y \uparrow+\infty} \frac{V(y)}{y}=0
$$

(см., например, [15, разд. 4]). В отличие от классических условий на функцию полезности, $V(y)$ может быть конечна только при больших значениях $y$ и не обязана быть конечной при всех $y>0$.

Нам будет удобно считать штрафную функцию $\gamma$ заданной на пространстве $\mathfrak{b} \mathfrak{a} \mathrm{c} \operatorname{dom} \gamma=\mathscr{Q}$. При этом целесообразно в определении функционала робастной полезности (1.1) брать инфимум по-прежнему по множеству $\mathscr{Q}$, чтобы избежать неопределенности вида $(-\infty)+(+\infty)$.

П р е д п о л о ж е н и е 2.2. Штрафная функиия $\gamma: \mathfrak{b a} \rightarrow[0,+\infty]$ - собственная выпуклая функиия, причем $\operatorname{dom} \gamma=: \mathscr{Q} \subseteq \mathfrak{P}$, и множеcmвo

$$
\left\{\frac{d \mathbf{Q}}{d \mathbf{P}}: \mathbf{Q} \in \mathscr{Q}, \gamma(\mathbf{Q}) \leqslant c\right\}
$$

замкнуто в $L^{1}$ и равномерно интегрируемо по отношению $\kappa \mathbf{P}$ для любого $c \geqslant 0$.

Поскольку множество (2.4) выпукло, его замкнутость в $L^{1}$ в сочетании с равномерной интегрируемостью эквивалентна его компактности в слабой топологии $\sigma\left(L^{1}, L^{\infty}\right)$ и, следовательно, компактности множества $\{\mathbf{Q} \in \mathscr{Q}: \gamma(\mathbf{Q}) \leqslant c\}$ в топологии $\sigma\left(\mathfrak{c a}, L^{\infty}\right)$ и $*$-слабой топологии $\sigma\left(\mathfrak{b a}, L^{\infty}\right)$. В частности, $\gamma$ полунепрерывна снизу в топологии $\sigma\left(\mathfrak{b a}, L^{\infty}\right)$.

Если штрафная функция $\gamma$ удовлетворяет предположению 2.2 и зафиксирована мера $\mathbf{Q} \in \mathscr{Q}$, то функция

$$
\gamma_{\mathbf{Q}}:=\gamma+\delta_{\{\mathbf{Q}\}}
$$

тоже, очевидно, удовлетворяет предположению 2.2 .

Приведем примеры штрафных функций, удовлетворяющих предположению 2.2. Пусть $f: \mathbf{R} \rightarrow \mathbf{R} \cup\{+\infty\}-$ собственная полунепрерывная снизу выпуклая функция с $\operatorname{dom} f \subseteq \mathbf{R}_{+}$, удовлетворяющая (4.3), $f(1) \geqslant 0$. Тогда предположение 2.2 будет выполнено, если

$$
\gamma(\mathbf{Q}):= \begin{cases}\mathscr{J}_{f}(\mathbf{Q}, \mathbf{P}), & \text { если } \mathbf{Q} \in \mathfrak{P} ; \\ +\infty & \text { в противном случае. }\end{cases}
$$


Еще один пример дает $\gamma:=\delta_{\mathscr{Q}}$, где $\mathscr{Q}=\left\{\mathbf{Q} \in \mathfrak{P}: \mathscr{J}_{f}(\mathbf{Q}, \mathbf{P}) \leqslant c\right\}, c \geqslant$ $f(1)$. Другие примеры можно найти, например, в работе [38].

Наши условия на штрафную функцию $\gamma$ хорошо известны в теории мер риска. А именно, функция $\rho: L^{\infty} \rightarrow \mathbf{R}$ является непрерьвной снизу выпуклой мерой риска тогда и только тогда, когда она представима в виде

$$
\rho(X)=-\min _{\mathbf{Q} \in \mathscr{Q}}\left[\mathbf{E}_{\mathbf{Q}} X+\gamma(\mathbf{Q})\right], \quad X \in L^{\infty},
$$

с функцией $\gamma$, удовлетворяющей предположению 2.2 (см. [19, теорема 5.2]).

П р е д п о л о ж е н и е 2.3. Множество терминальных капиталов $\mathscr{A}$ - выпуклое подмножество $L^{0}$, содержащее случайную величину $\xi_{0} \geqslant \varkappa$ для некоторого числа $\varkappa>0$.

По множеству $\mathscr{A}$ построим еще ряд множеств, играющих в дальнейшем существенную роль:

$$
\begin{gathered}
\mathscr{A}_{+}:=\mathscr{A} \cap L_{+}^{0}, \quad \mathscr{C}:=\left(\mathscr{A}-L_{+}^{0}\right) \cap L^{\infty}, \quad \mathscr{C}_{+}:=\mathscr{C} \cap L_{+}^{0}, \\
\overline{\mathscr{C}}_{+}-\text {замыкание } \mathscr{C}_{+} \text {в } L^{0}, \\
\mathscr{D}:=\left\{\eta \in L_{+}^{0}: \mathbf{E}_{\mathbf{P}} \eta \xi \leqslant 1 \text { для всех } \xi \in \mathscr{A}_{+}\right\} .
\end{gathered}
$$

Ясно, что $\mathscr{D} \subseteq L_{+}^{1}$, так как $\mathbf{E}_{\mathbf{P}} \eta \leqslant \varkappa^{-1}$ для любой $\eta \in \mathscr{D}$. Далее, заметим, что множества $\mathscr{C}_{+}, \overline{\mathscr{C}}_{+}, \mathscr{D}$ определяются множеством $\mathscr{A}_{+}$, в (2.6) множество $\mathscr{A}_{+}$можно заменить на $\mathscr{C}_{+}$и, по лемме Фату, на $\overline{\mathscr{C}}_{+}$. Множество $\overline{\mathscr{C}}_{+}$выпукло, телесно $\left(0 \leqslant \eta \leqslant \xi, \xi \in \overline{\mathscr{C}}_{+} \Rightarrow \eta \in \overline{\mathscr{C}}_{+}\right)$и замкнуто относительно сходимости по $\mathbf{P}$-вероятности. Поэтому по биполярной теореме Бранната-Шахермайера [5]

$$
\overline{\mathscr{C}}_{+}=\left\{\xi \in L_{+}^{0}: \mathbf{E}_{\mathbf{P}} \eta \xi \leqslant 1 \text { для всех } \eta \in \mathscr{D}\right\} .
$$

\section{2. Минимаксная теорема.}

Теорема 2.1. Пусть выполнены предположения 2.1-2.3. Тогда

$$
\sup _{\xi \in \mathscr{A}_{+}} \inf _{\mathbf{Q} \in \mathscr{Q}}\left[\mathbf{E}_{\mathbf{Q}} U(\xi)+\gamma(\mathbf{Q})\right]=\min _{\mathbf{Q} \in \mathscr{Q}} \sup _{\xi \in \mathscr{A}_{+}}\left[\mathbf{E}_{\mathbf{Q}} U(\xi)+\gamma(\mathbf{Q})\right] .
$$

Следует отметить отличие нашей формулировки от минимаксных теорем для робастной задачи, доказанных, скажем, в работах [41] и [38], где и в левой, и в правой частях минимаксного соотношения стоит верхняя грань по множеству $\mathscr{A}$, а не $\mathscr{A}_{+}$. Если, как предполагается в этих работах, существует такая мера $\mathbf{Q}_{0} \in \mathscr{Q}$, что $\mathbf{Q}_{0} \sim \mathbf{P}$, то замена $\mathscr{A}_{+}$ на $\mathscr{A}$, очевидно, не меняет левую часть (2.8), но может увеличить правую (если только не все меры из $\mathscr{Q}$ эквивалентны $\mathbf{P}$ ). Это объясняет, почему минимаксные теоремы в упомянутых работах доказаны при более сильных предположениях. Нам представляется более естественной наша постановка задачи. 
2.3. Максимизация полезности и арбитраж. Пусть $U(\infty):=$ $\sup _{x>0} U(x)$. Очевидно, что для монотонно неубывающей $U$

$$
\sup _{\xi \in \mathscr{A}_{+}} \mathbf{E}_{\mathbf{Q}} U(\xi) \leqslant U(\infty)
$$

и поэтому

$$
\sup _{\xi \in \mathscr{A}_{+}} \inf _{\mathbf{Q} \in \mathscr{Q}}\left[\mathbf{E}_{\mathbf{Q}} U(\xi)+\gamma(\mathbf{Q})\right] \leqslant U(\infty)+\inf _{\mathbf{Q} \in \mathscr{Q}} \gamma(\mathbf{Q})
$$

Достижимость равенства в (2.9) или в (2.10) делает задачу максимизации полезности в определенном смысле тривиальной и говорит о наличии той или иной формы арбитража. Легко видеть, что ситуация с равенством разнится в зависимости от того, ограничена сверху $U$ или нет. Мы приведем два предложения об условиях достижимости равенства в (2.9); условия равенства в (2.10) вытекают их этих предложений и теоремы 2.1. Предполагается, что $\mathbf{Q} \in \mathfrak{P}$.

Предложение 2.1. Пусть выполнено предположение 2.3. Тогда следующие утверждения эквивалентны:

(i) $\sup _{\xi \in \mathscr{A}_{+}} \mathbf{E}_{\mathbf{Q}} U(\xi)=\infty$ для любой неограниченной сверху функиии полезности $U$, удовлетворяющей предположению 2.1 ;

(ii) семейство $\mathscr{A}_{+}$неограничено по $\mathbf{Q}$-вероятности, т.е.

$$
\lim _{N \rightarrow \infty} \sup _{\xi \in \mathscr{A}_{+}} \mathbf{Q}(\xi>N)>0 .
$$

3 а м е ч а н и е 2.1 (ср. с предложением 4.19 в [20]). Пусть выполнено условие (ii) предложения 2.1. Как и в [20], несложно доказать следующее. Если функция полезности $U$ неограничена сверху, то верхняя грань в (2.9) либо не достигается, либо достигается на бесконечном множестве. Если функция полезности $U$ ограничена сверху и строго возрастает, то верхняя грань в $(2.9)$ не достигается.

Предложение 2.2. Пусть выполнено предположение 2.3. Следующие утверждения эквивалентны:

(i) $\sup _{\xi \in \mathscr{A}_{+}} \mathbf{E}_{\mathbf{Q}} U(\xi)=U(\infty)$ для любой ограниченной сверху функиии полезности $U$, удовлетворяющей предположению 2.1;

(ii) найдется такая ограниченная сверху функция полезности $U$, не равная тождественно константе на $(0,+\infty)$ и удовлетворяющая предположению 2.1 , что $\sup _{\xi \in \mathscr{A}_{+}} \mathbf{E}_{\mathbf{Q}} U(\lambda \xi)=U(\infty)$ для любого $\lambda>0$;

(iii) $\xi \mathbf{1}_{\{d \mathbf{Q} / d \mathbf{P}>0\}} \in \overline{\mathscr{C}}_{+}$для любой случайной величинь $\xi \in L_{+}^{0}$.

Забегая вперед, утверждение (ii) можно эквивалентно переформулировать так: для некоторой ограниченной сверху функции полезности $U$, не равной тождественно константе на $(0,+\infty), u(x)=U(\infty)$ для всех $x>0$, где функция $u$ задается соотношением $(2.11)$ с $\gamma=\delta_{\{\mathbf{Q}\}}$. 
2.4. Двойственная характеризация. Пусть множество $\mathscr{A}$ удовлетворяет предположению 2.3. Чтобы упростить обозначения, будем считать, что $\mathscr{A} \subseteq L_{+}^{0}$; в общем случае, надо везде заменить $\mathscr{A}$ на $\mathscr{A}_{+}$. Напомним, что множество $\mathscr{D}$ определено в (2.6). Для $x>0$ и $y \geqslant 0$ положим

$$
\mathscr{A}(x):=x \mathscr{A}, \quad \mathscr{D}(y):=y \mathscr{D} .
$$

Определим прямую и двойственную экстремальные задачи:

$$
\begin{aligned}
& u(x):=\sup _{\xi \in \mathscr{A}(x)} \inf _{\mathbf{Q} \in \mathscr{Q}}\left[\mathbf{E}_{\mathbf{Q}} U(\xi)+\gamma(\mathbf{Q})\right], \quad x>0, \\
& v(y):=\inf _{\eta \in \mathscr{D}(y), \mathbf{Q} \in \mathscr{Q}}\left[\mathbf{E}_{\mathbf{Q}} V\left(\frac{\eta}{d \mathbf{Q} / d \mathbf{P}}\right)+\gamma(\mathbf{Q})\right], \quad y \geqslant 0 .
\end{aligned}
$$

Напомним определение субдифференциала $\partial V(y)$ функции $V$ в точке $y \geqslant 0$ :

$$
\partial V(y):=\{z \in \mathbf{R}: V(t) \geqslant V(y)+z(t-y) \text { для всех } t \geqslant 0\},
$$

если $V(y)<+\infty$, и $\partial V(y):=\varnothing$ в противном случае. Аналогично, для $x \geqslant 0$

$$
\partial U(x):=\{z \in \mathbf{R}: U(t) \leqslant U(x)+z(t-x) \text { для всех } t \geqslant 0\} .
$$

Хорошо известно, что для $x, y \geqslant 0$

$$
U(x)=V(y)+x y \quad \Leftrightarrow \quad y \in \partial U(x) \quad \Leftrightarrow \quad x \in-\partial V(y),
$$

если $x>0$ или $U$ непрерывна справа в нуле; в противном случае имеет место только первая эквивалентность. Левая производная функции $U$ в точке $x>0$ обозначается $U_{-}^{\prime}(x)$.

Теорема 2.2. Пусть выполнены предположения $2.1-2.3, \mathscr{A} \subseteq L_{+}^{0}$, а функции $u, v$ задань соотношениями (2.11)-(2.12). Тогда имеют место следующие утверждения.

(i) Функиия $u(x), x>0$, принимает значения в $\mathbf{R} \cup\{+\infty\}$, является монотонно неубывающей и вогнутой.

(ii) Функция $v(y), y \geqslant 0$, принимает значения в $\mathbf{R} \cup\{+\infty\}$, является выпуклой, полунепрерывной снизу и монотонно невозрастающей. Инфимум в (2.12) достигается.

(iii) Ecли $v(y)=+\infty$ для всех $y \geqslant 0$, mо $u(x)=+\infty$ для всех $x>0$. Eсли $v(y)<+\infty$ для некоторого $y \geqslant 0$, то $u(x) \in \mathbf{R}$ при $x>0$.

(iv) Имеют место равенства

$$
\begin{array}{ll}
u(x)=\min _{y \geqslant 0}[v(y)+x y], & x>0, \\
v(y)=\sup _{x>0}[u(x)-x y], & y \geqslant 0 .
\end{array}
$$


(v) Зафиксируем $x>0$. Если минимум в (2.14) достигается на $y^{*}$, а минимум в (2.12) при $y=y^{*}-$ на паре $\left(\eta^{*}, \mathbf{Q}^{*}\right) \in \mathscr{D}\left(y^{*}\right) \times \mathscr{Q}$, mo

$$
u(x)=\sup _{\xi \in \mathscr{A}(x)}\left[\mathbf{E}_{\mathbf{Q}^{*}} U(\xi)+\gamma\left(\mathbf{Q}^{*}\right)\right] .
$$

Наоборот, если вьполнено (2.16) для $\mathbf{Q}^{*} \in \mathscr{Q}$, то найдутся такие $y^{*} \in$ $\mathbf{R}_{+} u \eta^{*} \in \mathscr{D}\left(y^{*}\right)$, что минимум в (2.14) достигается на $y^{*}$, а минимум в (2.12) при $y=y^{*}-$ на паре $\left(\eta^{*}, \mathbf{Q}^{*}\right)$.

(vi) Зафиксируем $x>0$. Пусть $u(x)<\infty$, минимум в (2.14) достигается на $y^{*}$, а минимум в (2.12) при $y=y^{*}-$ на паре $\left(\eta^{*}, \mathbf{Q}^{*}\right) \in$ $\mathscr{D}(y) \times \mathscr{Q}$. Тогда существование решения у задачи (2.11), т.е. такой случайной величинь $\xi^{*} \in \mathscr{A}(x)$, что $u(x)=\inf _{\mathbf{Q} \in \mathscr{Q}}\left[\mathbf{E}_{\mathbf{Q}} U\left(\xi^{*}\right)+\gamma(\mathbf{Q})\right]$, влечет соотношения

$$
\mathbf{E}_{\mathbf{P}} \xi^{*} \eta^{*}=x y^{*}
$$

$u \mathbf{P}-$ n.H.

$$
\frac{\eta^{*}}{d \mathbf{Q}^{*} / d \mathbf{P}} \in \partial U\left(\xi^{*}\right) н a\left\{\frac{d \mathbf{Q}^{*}}{d \mathbf{P}}>0\right\} \quad \text { и } \xi^{*} \eta^{*}=0 н a\left\{\frac{d \mathbf{Q}^{*}}{d \mathbf{P}}=0\right\} .
$$

Обратное утверждение верно частично: если (2.17) и (2.18) выполнень для некоторой $\xi^{*} \in \mathscr{A}(x)$, то $\xi^{*}$ есть решение задачи $(2.16)$, т.е. $и(x)=$ $\mathbf{E}_{\mathbf{Q}^{*}} U\left(\xi^{*}\right)+\gamma\left(\mathbf{Q}^{*}\right)$.

(vii) Ecлu $v \not \equiv+\infty$, mo

$$
\lim _{y \uparrow+\infty} \frac{v(y)}{y}=0 .
$$

(viii) Предположим, что функиия U удовлетворяет условию Инада в нуле, m.e.

$$
\lim _{x \downarrow 0} U_{-}^{\prime}(x)=+\infty .
$$

Предположим дополнительно, что $u(x)<\infty$ (в случае $U(\infty)=+\infty) u$ хотя бы для одной меры $\mathbf{Q} \in \arg \min \gamma$ не выполнены утверждения (i)(iii) предложения 2.2 (в случае $U(\infty)<+\infty)$. Тогда и удовлетворяет условию Инада в нуле.

(ix) Предположим, что функиия $U$ дифференцируема на $(0,+\infty)$ u удовлетворяет условию Инада в нуле, а функиия $\gamma$ строго выпукла на Q. Если $v(y)<+\infty$ для заданного у $>0$ и минимум в (2.12) достигается на парах $\left(\eta_{1}, \mathbf{Q}_{1}\right)$ и $\left(\eta_{2}, \mathbf{Q}_{2}\right)$, то $\mathbf{Q}_{1}=\mathbf{Q}_{2}$ и $\eta_{1}=\eta_{2} \mathbf{Q}_{1}$-п.н. Если функция и конечна, то она дифференцируема на $(0,+\infty)$. Только в случае ограниченности $U$ предположим дополнительно, что для мерь $\mathbf{Q}$, на которой достигается минимум функиии $\gamma$, не выполнены утверждения (i)-(iii) предложения 2.2. Тогда $v$ строго выпукла на $\{v<\infty\}$.

Как отмечалось во введении, данная теорема является обобщением теоремы 3.1 в [22], относящейся к стандартной задаче максимизации полезности. 
2.5. Случай всюду конечной функции полезности. Мы вкратце остановимся на случае, когда функция полезности конечна на всей числовой прямой, т.е. $U: \mathbf{R} \rightarrow \mathbf{R}-$ монотонно неубывающая вогнутая функция. Тогда, незначительно меняя доказательства и даже упрощая их, можно получить аналогичные результаты в предположении, что $\mathscr{A}-$ непустое выпуклое подмножество $L^{0}$ и каждая $\xi \in \mathscr{A}$ ограничена снизу (вместо предположения 2.3); предположение 2.2 остается в прежнем виде.

А именно, сохраняется минимаксная теорема:

$$
\sup _{\xi \in \mathscr{A}} \inf _{\mathbf{Q} \in \mathscr{Q}}\left[\mathbf{E}_{\mathbf{Q}} U(\xi)+\gamma(\mathbf{Q})\right]=\min _{\mathbf{Q} \in \mathscr{Q}} \sup _{\xi \in \mathscr{A}}\left[\mathbf{E}_{\mathbf{Q}} U(\xi)+\gamma(\mathbf{Q})\right] .
$$

Далее, зададим функцию $V$ соотношением

$$
V(y)=\sup _{x \in \mathbf{R}}[U(x)-x y], \quad y \in \mathbf{R} .
$$

По-прежнему, $V: \mathbf{R} \rightarrow \mathbf{R} \cup\{+\infty\}-$ собственная полунепрерывная снизу выпуклая функция, $\operatorname{dom} V \subseteq \mathbf{R}_{+}$,

$$
\inf _{y \in \mathbf{R}}[V(y)+x y]=U(x), \quad x \in \mathbf{R},
$$

но $V$ уже не будет невозрастающей функцией и

$$
\lim _{y \uparrow+\infty} \frac{V(y)}{y}=+\infty .
$$

Для формулировки аналога теоремы 2.2 в рассматриваемом случае естественно положить $\mathscr{A}(x):=x+\mathscr{A}$,

$$
u(x):=\sup _{\xi \in \mathscr{A}(x)} \inf _{\mathbf{Q} \in \mathscr{Q}}\left[\mathbf{E}_{\mathbf{Q}} U(\xi)+\gamma(\mathbf{Q})\right], \quad x \in \mathbf{R},
$$

и двойственную задачу определить как

$$
v(y):=\inf _{\mathbf{Q} \in \mathscr{Q}, \mathbf{Q}^{\prime} \in \mathfrak{P}, \mathbf{Q}^{\prime} \ll \mathbf{Q}}\left[\mathbf{E}_{\mathbf{Q}} V\left(y \frac{d \mathbf{Q}^{\prime}}{d \mathbf{Q}}\right)+y \sup _{\xi \in \mathscr{A}} \mathbf{E}_{\mathbf{Q}^{\prime}} \xi+\gamma(\mathbf{Q})\right], \quad y \geqslant 0 .
$$

Выражение упрощается, если дополнительно предположить, что $\mathscr{A}-$ конус.

Сформулируем теперь аналоги первых четырех утверждений теоремы 2.2. При сделанных предположениях функция $u(x), x \in \mathbf{R}$, принимает значения в $\mathbf{R} \cup\{+\infty\}$, является монотонно неубывающей и вогнутой, а функция $v(y), y \geqslant 0$, принимает значения в $\mathbf{R} \cup\{+\infty\}$, является выпуклой и полунепрерывной снизу. Инфимум в (2.22) достигается (ср. со следствием 2 в [15]). 
Далее, имеют место равенства

$$
\begin{array}{ll}
u(x)=\min _{y \geqslant 0}[v(y)+x y], & x \in \mathbf{R}, \\
v(y)=\sup _{x \in \mathbf{R}}[u(x)-x y], & y \geqslant 0 .
\end{array}
$$

В частности, если $v(y)=+\infty$ для всех $y \geqslant 0$, то $u(x)=+\infty$ для всех $x \in \mathbf{R}$. Если $v(y)<+\infty$ для некоторого $y \geqslant 0$, то $u(x) \in \mathbf{R}$ при $x \in \mathbf{R}$.

Следует, однако, отметить, что для функции полезности, конечной всюду, предположение об ограниченности снизу элементов $\mathscr{A}$ не является необходимым. Как уже отмечалось во введении, отказ от этого предположения значительно усложняет задачу и требует, при сохранении схемы рассуждений, значительных дополнительных усилий (см. $[27])$.

\section{3. Доказательства.}

Лемма 3.1. Пусть выполнень предположения 2.1-2.3, $\mathscr{A} \subseteq L^{\infty} u$ функция $U$ непрерывна справа в нуле. Тогда

$$
\sup _{\xi \in \mathscr{A}, U(\xi) \in L^{\infty}} \inf _{\mathbf{Q} \in \mathscr{Q}}\left[\mathbf{E}_{\mathbf{Q}} U(\xi)+\gamma(\mathbf{Q})\right]=\min _{\mu \in \mathfrak{b} \mathfrak{a}_{+}, \mathbf{Q} \in \mathscr{Q}}\left[\mathscr{J}_{V}(\mu, \mathbf{Q})+\sup _{\xi \in \mathscr{A}} \mu(\xi)+\gamma(\mathbf{Q})\right] .
$$

Д о к а з а т е л ь с т в о. Определим функции $\varphi$ и $\psi$ на $L^{\infty} \times L^{\infty}$ со значениями в $\mathbf{R} \cup\{+\infty\}$ соотношениями

$$
\varphi(\xi, \eta):=\delta_{\{(\xi, \eta): \eta \leqslant U(-\xi)\}}, \quad \psi(\xi, \eta):=\delta_{\mathscr{A}}(\xi)+\sup _{\mathbf{Q} \in \mathscr{Q}}\left[\mathbf{E}_{\mathbf{Q}} \eta-\gamma(\mathbf{Q})\right] .
$$

Пусть $\widetilde{u}$ обозначает левую часть (3.1). Справедлива следующая цепочка равенств:

$$
\begin{aligned}
-\widetilde{u} & =\inf _{\xi \in \mathscr{A}, U(\xi) \in L^{\infty}} \sup _{\mathbf{Q} \in \mathscr{Q}}\left[-\mathbf{E}_{\mathbf{Q}} U(\xi)-\gamma(\mathbf{Q})\right] \\
& \stackrel{(*)}{=} \inf _{\xi \in \mathscr{A}, \eta \in L^{\infty}, \eta \geqslant-U(\xi)} \sup _{\mathbf{Q} \in \mathscr{Q}}\left[\mathbf{E}_{\mathbf{Q}} \eta-\gamma(\mathbf{Q})\right] \\
& =\inf _{(\xi, \eta) \in L^{\infty} \times L^{\infty}}[\varphi(-\xi,-\eta)+\psi(\xi, \eta)],
\end{aligned}
$$

где равенство, помеченное $(*)$, использует тот факт, что для $\xi \in L^{\infty}$ случайная величина $-U(\xi)$ всегда ограничена снизу константой.

Ясно, что $\varphi$ и $\psi-$ выпуклые функции, $\operatorname{dom} \psi=\mathscr{A} \times L^{\infty}$. Возьмем $\varepsilon \in(0, \varkappa)$. Тогда случайные величины $|U(\xi)|$, где $\xi \in L^{\infty},\left\|\xi-\xi_{0}\right\|_{L^{\infty}}<\varepsilon$, равномерно ограничены, скажем, числом $b$. Если взять $\eta_{0}:=b+\varepsilon$, то $\varphi$ будет непрерывной по норме в точке $\left(-\xi_{0},-\eta_{0}\right)$. Поэтому применима теорема двойственности Фенхеля-Рокафеллара (см. п. 4.1), согласно которой

$$
\widetilde{u}=\min _{\mu, \nu \in \mathfrak{b} \mathfrak{a}}\left[\varphi^{*}(\mu, \nu)+\psi^{*}(\mu, \nu)\right]
$$


Согласно [15] (см. п. 4.2 ниже), $\varphi^{*}(\mu, \nu)=\mathscr{J}_{V}(\mu, \nu)$ и $\operatorname{dom} \varphi^{*} \subseteq$ $\mathfrak{b} \mathfrak{a}_{+} \times \mathfrak{b} \mathfrak{a}_{+}$. Поскольку второе слагаемое в определении $\psi$ есть преобразование Фенхеля выпуклой и полунепрерывной снизу (в $*$-слабой топологии) функции $\gamma: \mathfrak{b a} \rightarrow \mathbf{R} \cup\{+\infty\}$, имеем $\psi^{*}(\mu, \nu)=\sup _{\xi \in \mathscr{A}} \mu(\xi)+\gamma(\nu)$. Объединяя сказанное, приходим к равенству (3.1). Лемма доказана.

Лемма 3.2. Пусть выполнены предположения 2.1-2.3. Тогда

$$
\begin{aligned}
\sup _{\xi \in \mathscr{A}_{+}} & \inf _{\mathbf{Q} \in \mathscr{Q}}\left[\mathbf{E}_{\mathbf{Q}} U(\xi)+\gamma(\mathbf{Q})\right] \\
& =\min _{\mu \in \mathfrak{b} \mathfrak{a}_{+}, \mathbf{Q} \in \mathscr{Q}}\left[\mathscr{J}_{V}(\mu, \mathbf{Q})+\sup _{\xi \in \mathscr{C}} \mu(\xi)+\gamma(\mathbf{Q})\right] \\
& =\min _{\mu \in \mathfrak{b} \mathfrak{a}_{+}, \mathbf{Q} \in \mathscr{Q}}\left[\mathscr{J}_{V}(\mu, \mathbf{Q})+\sup _{\xi \in \mathscr{C}_{+}} \mu(\xi)+\gamma(\mathbf{Q})\right] \\
& =\min _{\mu \in \mathfrak{c a}+, \mathbf{Q} \in \mathscr{Q}}\left[\mathscr{J}_{V}(\mu, \mathbf{Q})+\sup _{\xi \in \mathscr{C}_{+}} \mu(\xi)+\gamma(\mathbf{Q})\right] .
\end{aligned}
$$

3 а м е ч а н и е 3.1 . Очевидно, что в (3.5) множество $\mathscr{C}_{+}$можно заменить на $\mathscr{A}_{+}$. Однако нельзя, по аналогии с $(3.3)$ и $(3.4)$, заменить в $(3.5) \mathscr{C}_{+}$на $\mathscr{C}$.

В связи с предыдущим замечанием отметим, что значения функционала $\sup _{\xi \in \mathscr{C}} \mu(\xi)$, стоящего в (3.3), зависят от всего множества $\mathscr{A}$, а не только от его пересечения $\mathscr{A}_{+}$с положительным конусом. Тем не менее, из леммы 3.2 следует, что значение минимума из (3.3) зависит только от $\mathscr{A}_{+}$.

Разумеется, в (3.3)-(3.5), используя результаты из п. 4.2, $V$ дивергенцию $\mathscr{J}_{V}(\mu, \mathbf{Q})$ можно записать через математическое ожидание по мере $\mathbf{Q}$ в виде, сходном с определением (2.12) функции $v$. Мы этого не делаем, поскольку в доказательстве теоремы 2.2 при исследовании свойств функции $v$ нам будет удобнее, наоборот, записать выражение для $v$ в терминах $V$-дивергенции.

Док аз а т ель с т в о л е м м ы 3.2. Применяя лемму 3.1 к функции полезности $\widetilde{U}$ (см. (2.2)), штрафной функции $\gamma$ и множеству $\mathscr{C}(x):=x+\mathscr{C}$ в роли $\mathscr{A}$, где $x-$ число, $x>-\varkappa$, получим

$$
\begin{aligned}
\widetilde{u}(x) & :=\sup _{\xi \in \mathscr{C}(x), \tilde{U}(\xi) \in L^{\infty}} \inf _{\mathbf{Q} \in \mathscr{Q}}\left[\mathbf{E}_{\mathbf{Q}} \widetilde{U}(\xi)+\gamma(\mathbf{Q})\right] \\
& =\min _{\mu \in \mathfrak{b} \mathfrak{a}_{+}, \mathbf{Q} \in \mathscr{Q}}\left[\mathscr{J}_{V}(\mu, \mathbf{Q})+\sup _{\xi \in \mathscr{C}} \mu(x+\xi)+\gamma(\mathbf{Q})\right] \\
& =\min _{\substack{y \geqslant 0, \mathbf{Q} \in \mathscr{Q} \\
\mu \in \mathfrak{b} \mathfrak{a}_{+}, \mu(\Omega)=1}}\left[\mathscr{J}_{V}(y \mu, \mathbf{Q})+x y+y \sup _{\xi \in \mathscr{C}} \mu(\xi)+\gamma(\mathbf{Q})\right] .
\end{aligned}
$$

Из (3.7) следует, что функция $\widetilde{u}(x), x>-\varkappa$, вогнута и монотонно не убывает; легко также видеть, что она не принимает значения $-\infty$. Значит, она либо тождественно равна $+\infty$, либо всюду конечна. В обоих случаях она непрерывна. 
Далее, обозначим выражение (3.2) через $u$. Для любого $\varepsilon>0$

$$
u \leqslant \inf _{\mathbf{Q} \in \mathscr{Q}} \sup _{\xi \in \mathscr{A}_{+}}\left[\mathbf{E}_{\mathbf{Q}} U(\xi)+\gamma(\mathbf{Q})\right] \leqslant \inf _{\mathbf{Q} \in \mathscr{Q}} \sup _{\xi \in \mathscr{A}_{+}}\left[\mathbf{E}_{\mathbf{Q}} \widetilde{U}(\varepsilon+\xi)+\gamma(\mathbf{Q})\right] .
$$

Для $\xi \in \mathscr{A}_{+}$положим $\xi_{n}:=\xi \wedge n$, тогда $\xi_{n} \in \mathscr{C}$ и $\widetilde{U}\left(\varepsilon+\xi_{n}\right) \geqslant \widetilde{U}(\varepsilon)>-\infty$, поэтому по теореме о монотонной сходимости для любой $\mathbf{Q} \in \mathscr{Q}$

$$
\mathbf{E}_{\mathbf{Q}} \widetilde{U}(\varepsilon+\xi)=\lim _{n} \mathbf{E}_{\mathbf{Q}} \widetilde{U}\left(\varepsilon+\xi_{n}\right) \leqslant \sup _{\eta \in \mathscr{C}(\varepsilon), \tilde{U}(\eta) \in L^{\infty}} \mathbf{E}_{\mathbf{Q}} \widetilde{U}(\eta),
$$

откуда

$$
\begin{aligned}
u & \leqslant \inf _{\mathbf{Q} \in \mathscr{Q}} \sup _{\eta \in \mathscr{C}(\varepsilon), \tilde{U}(\eta) \in L^{\infty}}\left[\mathbf{E}_{\mathbf{Q}} \widetilde{U}(\eta)+\gamma(\mathbf{Q})\right] \\
& =\inf _{\mathbf{Q} \in \mathscr{Q}} \min _{\mu \in \mathfrak{b}_{+}}\left[\mathscr{J}_{V}(\mu, \mathbf{Q})+\sup _{\xi \in \mathscr{C}} \mu(\varepsilon+\xi)+\gamma(\mathbf{Q})\right]=\widetilde{u}(\varepsilon),
\end{aligned}
$$

где в обоих равенствах мы воспользовались соотношением (3.6), причем в первом случае - со штрафной функцией $\gamma_{\mathbf{Q}}($ см. $(2.5))$.

С другой стороны, пусть $0<\varepsilon<\varkappa$ и $\xi+\varepsilon \in \mathscr{C}$. Тогда найдется такая случайная величина $\eta \in \mathscr{A}$, что $\xi+\varepsilon \leqslant \eta$, причем $U(\eta) \geqslant U(\xi+\varepsilon) \geqslant \widetilde{U}(\xi)$; в частности, из $\widetilde{U}(\xi) \in L^{\infty}$ следует, что $\eta \geqslant 0$. Поэтому

$$
\begin{aligned}
\widetilde{u}(-\varepsilon) & =\sup _{\xi+\varepsilon \in \mathscr{C}, \tilde{U}(\xi) \in L^{\infty}} \inf _{\mathbf{Q} \in \mathscr{Q}}\left[\mathbf{E}_{\mathbf{Q}} \widetilde{U}(\xi)+\gamma(\mathbf{Q})\right] \\
& \leqslant \sup _{\eta \in \mathscr{A}_{+}} \inf _{\mathbf{Q} \in \mathscr{Q}}\left[\mathbf{E}_{\mathbf{Q}} U(\eta)+\gamma(\mathbf{Q})\right]=u .
\end{aligned}
$$

Из (3.8), (3.9) и непрерывности $\widetilde{u}$ следует, что $u=\widetilde{u}(0)$, и равенство (3.3) есть следствие (3.6).

Если применить доказанное утверждение для $\mathscr{A}_{+}$в роли $\mathscr{A}$, то $\mathscr{C}=\left\{\xi \in L^{\infty}:\right.$ найдется такая $\eta \in \mathscr{A}_{+}$, что $\left.\xi \leqslant \eta\right\}$ обладает свойством: если $\xi \in \mathscr{C}$, то $\max \{\xi, 0\} \in \mathscr{C}$. Поэтому в рассматриваемом случае (когда $\left.\mathscr{A}=\mathscr{A}_{+}\right) \sup _{\xi \in \mathscr{C}} \mu(\xi)=\sup _{\xi \in \mathscr{C}_{+}} \mu(\xi)$ для $\mu \in \mathfrak{b a}_{+}$, и верно (3.4). Равенство (3.5) верно в силу того, что для $\mu \in \mathfrak{b a}_{+}$

$$
\mathscr{J}_{V}(\mu, \mathbf{Q})=\mathscr{J}_{V}\left(\mu^{\mathrm{r}}, \mathbf{Q}\right) \quad \text { и } \quad \sup _{\xi \in \mathscr{C}_{+}} \mu(\xi) \geqslant \sup _{\xi \in \mathscr{C}_{+}} \mu^{\mathrm{r}}(\xi)
$$

Лемма доказана.

Д о к а з а т е л ь с т в о т е о р е м ы 2.1. Применяя лемму 3.2 с теми же $U$ и $\mathscr{A}$ и функцией полезности $\gamma_{\mathbf{Q}}$, см. $(2.5)$, получаем для фиксированной $\mathbf{Q} \in \mathscr{Q}$, что

$$
\sup _{\xi \in \mathscr{A}_{+}}\left[\mathbf{E}_{\mathbf{Q}} U(\xi)+\gamma(\mathbf{Q})\right]=\min _{\mu \in \mathfrak{b} \mathfrak{a}_{+}}\left[\mathscr{J}_{V}(\mu, \mathbf{Q})+\sup _{\xi \in \mathscr{C}} \mu(\xi)+\gamma(\mathbf{Q})\right] .
$$


Сравнивая это равенство с (3.3), немедленно приходим к минимаксному соотношению.

Д ок аз ательств о т е о ремы 2.2. Мы начнем с доказательства утверждения (ii) о свойствах функции $v$. Доказать достижимость инфимума в (2.12) можно с помощью стандартных аргументов типа теоремы Комлоша (см., например, доказательство леммы 3.3 в [22] для стандартной задачи или доказательства леммы 3.7 в [41] и леммы 4.3 в [38] для робастной задачи). Но мы последовательно будем использовать другой подход, основанный на представлении функции $v$ в терминах $V$-дивергенции (см. (3.10) ниже) и общих свойствах $V$-дивергенции.

Положим

$$
\mathscr{R}:=\left\{\mu \in \mathfrak{b a}_{+}: \mu(\xi) \leqslant 1 \text { для всех } \xi \in \mathscr{C}_{+}\right\} .
$$

Заметим, что $\mu(\Omega) \leqslant \varkappa^{-1}$ для $\mu \in \mathscr{R}$, поэтому по теореме БанахаАлаоглу $\mathscr{R}$ есть компакт в $*$-слабой топологии $\sigma\left(\mathfrak{b} \mathfrak{a}, L^{\infty}\right)$. Легко видеть, что

$$
v(y)=\inf _{\mu \in \mathscr{R}, \mathbf{Q} \in \mathscr{Q}}\left[\mathscr{J}_{V}(y \mu, \mathbf{Q})+\gamma(\mathbf{Q})\right], \quad y \geqslant 0 .
$$

Действительно, $\mathscr{J}_{V}(y \mu, \mathbf{Q})=\mathscr{J}_{V}\left(y \mu^{\mathrm{r}}, \mathbf{Q}\right)=\mathbf{E}_{\mathbf{Q}} V\left(y \frac{d \mu^{\mathrm{r}} / d \mathbf{P}}{d \mathbf{Q} / d \mathbf{P}}\right)$ и

$$
\left\{\frac{d \mu^{\mathrm{r}}}{d \mathbf{P}}: \mu \in \mathscr{R}\right\}=\mathscr{D}
$$

откуда и следует совпадение правых частей (2.12) и (3.10).

Пусть $v(y)<+\infty$ для заданного $y$. Поскольку функции $\mathfrak{b} \mathfrak{a} \times \mathfrak{b} \mathfrak{a} \ni$ $(\mu, \nu) \rightsquigarrow \mathscr{J}_{V}(y \mu, \nu)$ и $\gamma$ полунепрерывны снизу, а множество $\mathscr{R}$ компактно в соответствующих *-слабых топологиях и $\mathscr{J}_{V}(y \mu, \mathbf{Q}) \geqslant V(y \mu(\Omega)) \geqslant$ $V\left(y \varkappa^{-1}\right)$ для $\mathbf{Q} \in \mathscr{Q}$ и $\mu \in \mathscr{R}$, то множества

$$
\left\{(\mu, \nu) \in \mathfrak{b a} \times \mathfrak{b a}: \mathscr{J}_{V}(y \mu, \nu)+\delta_{\mathscr{R}}(\mu)+\gamma(\nu) \leqslant c\right\}
$$

для всех $c \in \mathbf{R}$ являются компактами в $*$-слабой топологии $\sigma(\mathfrak{b a} \times$ $\left.\mathfrak{b} \mathfrak{a}, L^{\infty} \times L^{\infty}\right)$ ввиду соответствующего свойства функции $\gamma$ из предположения 2.2. Значит, нижняя грань в (3.10) достигается, скажем, на паре $\left(\mu^{*}, \mathbf{Q}^{*}\right)$. Тогда в $(2.12)$ инфимум достигается на паре $\left(y d \mu^{* r} / d \mathbf{P}, \mathbf{Q}^{*}\right)$.

Проверим выпуклость $v$. Пусть $0 \leqslant y_{1}<y_{2}<+\infty, \alpha \in(0,1)$, а меры $\mu_{i} \in \mathscr{R}$ и $\mathbf{Q}_{i} \in \mathscr{Q}$ таковы, что $v\left(y_{i}\right)=\mathscr{J}_{V}\left(y_{i} \mu_{i}, \mathbf{Q}_{i}\right)+\gamma\left(\mathbf{Q}_{i}\right)$, $i=1,2$. Положим $y:=\alpha y_{1}+(1-\alpha) y_{2}, \mu:=\alpha y_{1} y^{-1} \mu_{1}+(1-\alpha) y_{2} y^{-1} \mu_{2}$, $\mathbf{Q}:=\alpha \mathbf{Q}_{1}+(1-\alpha) \mathbf{Q}_{2}$, тогда $\mu \in \mathscr{R}$ и $\mathbf{Q} \in \mathscr{Q}$. В силу выпуклости $V$-дивергенции

$$
\begin{aligned}
\mathscr{J}_{V}(y \mu, \mathbf{Q}) & =\mathscr{J}_{V}\left(\alpha y_{1} \mu_{1}+(1-\alpha) y_{2} \mu_{2}, \alpha \mathbf{Q}_{1}+(1-\alpha) \mathbf{Q}_{2}\right) \\
& \leqslant \alpha \mathscr{J}_{V}\left(y_{1} \mu_{1}, \mathbf{Q}_{1}\right)+(1-\alpha) \mathscr{J}_{V}\left(y_{2} \mu_{2}, \mathbf{Q}_{2}\right),
\end{aligned}
$$


откуда с учетом выпуклости функции $\gamma$ получаем неравенство $v(y) \leqslant$ $\alpha v\left(y_{1}\right)+(1-\alpha) v\left(y_{2}\right)$.

Далее, из неравенства $\eta \leqslant U(-\xi)$ для $\xi, \eta \in L^{\infty}$ следует, что $\xi \leqslant 0$. Поэтому для любых $\mu_{1}, \mu_{2}, \nu \in \mathfrak{b a}_{+}$

$$
\begin{aligned}
\mathscr{J}_{V}\left(\mu_{1}+\mu_{2}, \nu\right) & =\sup _{\xi, \eta \in L^{\infty}, \eta \leqslant U(-\xi)}\left[\left(\mu_{1}+\mu_{2}\right)(\xi)+\nu(\eta)\right] \\
& \leqslant \sup _{\xi, \eta \in L^{\infty}, \eta \leqslant U(-\xi)}\left[\mu_{1}(\xi)+\nu(\eta)\right] \\
& =\mathscr{J}_{V}\left(\mu_{1}, \nu\right) .
\end{aligned}
$$

Поэтому функция $v$ не возрастает.

В частности, $\{y \geqslant 0: v(y)<\infty\}$ есть интервал в $\mathbf{R}_{+}$, либо пустой, либо вида $(l,+\infty)$ или $[l,+\infty)$. Пусть $v \not \equiv+\infty$. В силу выпуклости функция $v$ непрерывна в $(l,+\infty)$, поэтому для доказательства полунепрерывности снизу функции $v$ достаточно проверить ее полунепрерывность снизу в точке $l$. Положим $c:=\lim _{y \downarrow l} v(y)$ и определим $K(y):=\left\{(\mu, \mathbf{Q}) \in \mathscr{R} \times \mathscr{Q}: \mathscr{J}_{V}(y \mu, \mathbf{Q})+\gamma(\mathbf{Q}) \leqslant c\right\}$. Тогда множества $K(l+1 / n)$ непусты, убывают по $n$ в силу (3.12) и, так же как и в (3.11), являются компактами в $*$-слабой топологии $\sigma\left(\mathfrak{b a} \times \mathfrak{b a}, L^{\infty} \times L^{\infty}\right)$. Поэтому их пересечение непусто. Из полунепрерывности снизу $V$-дивергенции следует $\bigcap_{n} K(l+1 / n) \subseteq K(l)$, что и доказывает полунепрерывность снизу функции $v$.

Докажем теперь утверждение (vii). Из соотношения (2.3) и неравенства $v(y) \geqslant V\left(y \varkappa^{-1}\right), y \geqslant 0$, следует, что

$$
\liminf _{y \uparrow+\infty} \frac{v(y)}{y} \geqslant 0 .
$$

С другой стороны,

$$
\limsup _{y \uparrow+\infty} \frac{v(y)}{y} \leqslant 0,
$$

так как $v$ не возрастает и не равна тождественно $+\infty$.

Далее, по лемме 3.2

$$
u(x)=\min _{\eta \in L_{+}^{1}, \mathbf{Q} \in \mathscr{Q}}\left[\mathbf{E}_{\mathbf{Q}} V\left(\frac{\eta}{d \mathbf{Q} / d \mathbf{P}}\right)+x \sup _{\xi \in \mathscr{C}_{+}} \mathbf{E}_{\mathbf{P}} \xi \eta+\gamma(\mathbf{Q})\right] .
$$

Положим

$$
\widehat{\mathscr{D}}(y):=\left\{\eta \in L_{+}^{0}: \sup _{\xi \in \mathscr{C}_{+}} \mathbf{E}_{\mathbf{P}} \eta \xi=y\right\} .
$$

Возможны два случая: $\widehat{\mathscr{D}}(y)=\varnothing$ для любого $y>0$ и $\widehat{\mathscr{D}}(y) \neq \varnothing$ для любого $y>0$. В первом случае получаем, что $u(x)=V(0)+\inf _{\mathbf{Q} \in \mathscr{Q}} \gamma(\mathbf{Q})=$ $v(y)$ для всех $x>0$ и $y \geqslant 0$, и $(2.14)$ верно. Во втором случае $\widehat{\mathscr{D}}(0)=\{0\}=\mathscr{D}(0)$, а при $y>0 \widehat{\mathscr{D}}(y) \subseteq \mathscr{D}(y)$ и для любой $\eta \in \mathscr{D}(y)$ 
найдется такая $\eta^{\prime} \in \widehat{\mathscr{D}}(y)$, что $\eta^{\prime} \geqslant \eta$, поэтому в определении (2.12) функции $v$ множество $\mathscr{D}(y)$ можно заменить на $\widehat{\mathscr{D}}(y)$. Следовательно,

$$
\begin{aligned}
u(x) & =\min _{y \geqslant 0} \inf _{\eta \in \widehat{\mathscr{D}}(y), \mathbf{Q} \in \mathcal{Q}}\left[\mathbf{E}_{\mathbf{Q}} V\left(\frac{\eta}{d \mathbf{Q} / d \mathbf{P}}\right)+x y+\gamma(\mathbf{Q})\right] \\
& =\min _{y \geqslant 0}[v(y)+x y] .
\end{aligned}
$$

Таким образом, мы доказали (2.14). Отсюда также вытекают утверждения (i) и (iii) теоремы.

Докажем соотношение (2.15). В силу утверждения (iii) можно ограничиться случаем $v \not \equiv+\infty$. Положим

$$
\bar{u}(x):=\inf _{y \geqslant 0}[v(y)+x y], \quad x \in \mathbf{R} .
$$

По теореме Фенхеля-Моро

$$
v(y)=\sup _{x \in \mathbf{R}}[\bar{u}(x)-x y], \quad y \geqslant 0 .
$$

Теперь заметим, что $\bar{u}(x)=-\infty$ для всех $x<0$ в силу утверждения (vii), поэтому в (3.13) можно брать супремум по $x \geqslant 0$ и, значит, по $x>0$ в силу монотонности $\bar{u}$. Поскольку $\bar{u}(x)$ совпадает с $u(x)$ при $x>0$ в силу (2.14), правые части (3.13) и (2.15) совпадают.

Докажем утверждение $(\mathrm{v})$. Пусть $u(x)=v\left(y^{*}\right)+x y^{*}, y^{*} \geqslant 0$, $v\left(y^{*}\right)=\mathbf{E}_{\mathbf{Q}^{*}} V\left(\eta^{*}\left(d \mathbf{Q}^{*} / d \mathbf{P}\right)^{-1}\right)+\gamma\left(\mathbf{Q}^{*}\right), \eta^{*} \in \mathscr{D}\left(y^{*}\right), \mathbf{Q}^{*} \in \mathscr{Q}$. Возьмем $\mu^{*}=\left(\eta^{*} / y^{*}\right) \cdot \mathbf{P}$ в случае $y^{*}>0$ и $\mu^{*}=0$, если $y^{*}=0$. Тогда $\mu^{*} \in \mathscr{R}$ и $v\left(y^{*}\right)=\mathscr{J}_{V}\left(y^{*} \mu^{*}, \mathbf{Q}^{*}\right)+\gamma\left(\mathbf{Q}^{*}\right)$, поэтому

$$
\begin{aligned}
u(x) & =\mathscr{J}_{V}\left(y^{*} \mu^{*}, \mathbf{Q}^{*}\right)+x y^{*}+\gamma\left(\mathbf{Q}^{*}\right) \\
& \geqslant \inf _{\mu \in \mathfrak{c} \mathfrak{a}_{+}}\left[\mathscr{J}_{V}\left(\mu, \mathbf{Q}^{*}\right)+x \sup _{\xi \in \mathscr{C}_{+}} \mu(\xi)+\gamma\left(\mathbf{Q}^{*}\right)\right] \\
& =\sup _{\xi \in \mathscr{A}(x)}\left[\mathbf{E}_{\mathbf{Q}^{*}} U(\xi)+\gamma\left(\mathbf{Q}^{*}\right)\right],
\end{aligned}
$$

где в последнем равенстве мы воспользовались соотношением (3.5) с $\mathscr{A}(x)$ в роли $\mathscr{A}$ и со штрафной функцией $\gamma_{\mathbf{Q}^{*}}$. Обратное неравенство очевидно.

В обратную сторону, пусть верно (2.16). Вновь пользуясь (3.5) со штрафной функцией $\gamma_{\mathbf{Q}^{*}}$, найдем такую $\bar{\mu} \in \mathfrak{c a}_{+}$, что

$$
u(x)=\mathscr{J}_{V}\left(\bar{\mu}, \mathbf{Q}^{*}\right)+x \sup _{\xi \in \mathscr{C}_{+}} \bar{\mu}(\xi)+\gamma\left(\mathbf{Q}^{*}\right)
$$

Пусть $y^{*}:=\sup _{\xi \in \mathscr{C}_{+}} \bar{\mu}(\xi)$, тогда предыдущее равенство можно переписать в виде $u(x)=\mathscr{J}_{V}\left(y^{*} \mu^{*}, \mathbf{Q}^{*}\right)+x y^{*}+\gamma\left(\mathbf{Q}^{*}\right)$, где $\mu^{*} \in \mathscr{R}$ такова, что $y^{*} \mu^{*}=\bar{\mu}$. Так как $v\left(y^{*}\right) \leqslant \mathscr{J}_{V}\left(y^{*} \mu^{*}, \mathbf{Q}^{*}\right)+\gamma\left(\mathbf{Q}^{*}\right)$, имеем $u(x) \geqslant v\left(y^{*}\right)+x y^{*}$, 
что в сочетании с (2.14) дает два равенства $u(x)=v\left(y^{*}\right)+x y^{*}$ и $v\left(y^{*}\right)=\mathscr{J}_{V}\left(y^{*} \mu^{*}, \mathbf{Q}^{*}\right)+\gamma\left(\mathbf{Q}^{*}\right)=\mathscr{J}_{V}\left(y^{*} \mu^{* r}, \mathbf{Q}^{*}\right)+\gamma\left(\mathbf{Q}^{*}\right)$, т.е. минимум в $(2.12)$ достигается на $\left(y^{*} d \mu^{* r} / d \mathbf{P}, \mathbf{Q}^{*}\right)$.

Теперь докажем пункт (vi). Пусть $\xi^{*} \in \mathscr{A}(x)$. Из определения $V$ следует, что на $\left\{d \mathbf{Q}^{*} / d \mathbf{P}>0\right\}$

$$
U\left(\xi^{*}\right)+\gamma\left(\mathbf{Q}^{*}\right) \leqslant V\left(\frac{\eta^{*}}{d \mathbf{Q}^{*} / d \mathbf{P}}\right)+\frac{\xi^{*} \eta^{*}}{d \mathbf{Q}^{*} / d \mathbf{P}}+\gamma\left(\mathbf{Q}^{*}\right)
$$

Беря математическое ожидание по мере $\mathbf{Q}^{*}$ от обеих частей, получим

$$
\begin{aligned}
\mathbf{E}_{\mathbf{Q}^{*}} U\left(\xi^{*}\right)+\gamma\left(\mathbf{Q}^{*}\right) & \leqslant \mathbf{E}_{\mathbf{Q}^{*}} V\left(\frac{\eta^{*}}{d \mathbf{Q}^{*} / d \mathbf{P}}\right)+\mathbf{E}_{\mathbf{Q}^{*}} \frac{\xi^{*} \eta^{*}}{d \mathbf{Q}^{*} / d \mathbf{P}}+\gamma\left(\mathbf{Q}^{*}\right) \\
& =v\left(y^{*}\right)+\mathbf{E}_{\mathbf{P}}\left(\xi^{*} \eta^{*} \mathbf{1}_{\left\{d \mathbf{Q}^{*} / d \mathbf{P}>0\right\}}\right) \leqslant v\left(y^{*}\right)+x y^{*} .
\end{aligned}
$$

Если $\xi^{*}$ есть решение задачи (2.11), то, с учетом (2.16), левая часть (3.15) равна $u(x)$. Значит, оба неравенства в (3.15) суть равенства, и, следовательно, в (3.14) имеет место равенство $\mathbf{Q}^{*}$-п.н., т.е. $\mathbf{P}$-п.н. на множестве $\left\{d \mathbf{Q}^{*} / d \mathbf{P}>0\right\}$. Отсюда и из (2.13) следуют (2.17) и (2.18). Если же (2.17) и (2.18) имеют место, то в (3.14) левая и правая часть равны $\mathbf{P}$-п.н. на $\left\{d \mathbf{Q}^{*} / d \mathbf{P}>0\right\}$, и мы приходим к равенствам вместо неравенств в (3.15). Значит, $u(x)=\mathbf{E}_{\mathbf{Q}^{*}} U\left(\xi^{*}\right)+\gamma\left(\mathbf{Q}^{*}\right)$.

Перейдем к доказательству (viii). По предложению 4 в [15], выполнение условия Инада (2.19) означает строгое убывание функции $V$. Соответственно, невыполнение условия Инада в нуле для функции $u$ означает, что либо $u \equiv+\infty$ (что исключено по условию), либо $u(x)<+\infty$ для всех $x>0$ и функция $v$ конечна и постоянна на некотором интервале, а именно на интервале $[\beta,+\infty)$, где $\beta:=\lim _{x \downarrow 0} u_{-}^{\prime}(x)$. Предположим, что функция $v$ ведет себя именно так.

Зафиксируем $y>\beta$. Пусть минимум в (2.12) достигается на паре $\left(\eta^{*}, \mathbf{Q}^{*}\right)$, т.е.

$$
v(y)=\mathbf{E}_{\mathbf{Q}^{*}} V\left(\frac{\eta^{*}}{d \mathbf{Q}^{*} / d \mathbf{P}}\right)+\gamma\left(\mathbf{Q}^{*}\right), \quad \mathbf{Q}^{*} \in \mathscr{Q}, \quad \eta^{*} \in \mathscr{D}(y) .
$$

Так как $\lambda \eta^{*} \in \mathscr{D}(\lambda y)$ и $v(\lambda y)=v(y)$ при $\lambda>1$, то

$$
\mathbf{E}_{\mathbf{Q}^{*}} V\left(\frac{\lambda \eta^{*}}{d \mathbf{Q}^{*} / d \mathbf{P}}\right) \geqslant \mathbf{E}_{\mathbf{Q}^{*}} V\left(\frac{\eta^{*}}{d \mathbf{Q}^{*} / d \mathbf{P}}\right)
$$

по определению $v(\lambda y)$. С другой стороны, $V\left(\frac{\lambda \eta^{*}}{d \mathbf{Q}^{*} / d \mathbf{P}}\right) \leqslant V\left(\frac{\eta^{*}}{d \mathbf{Q}^{*} / d \mathbf{P}}\right)$. В силу строгой монотонности $V$ комбинация двух последних неравенств возможна лишь в случае, когда $\eta^{*}=0 \mathbf{Q}^{*}$-п.н. Значит, $v(y)=V(0)+$ $\gamma\left(\mathbf{Q}^{*}\right)$, в частности, $V(0)=U(\infty)<\infty$ и любая $\eta \in \mathscr{D}(y)$ (и, значит, 
любая $\eta \in \mathscr{D})$ удовлетворяет $\eta=0 \mathbf{Q}^{*}$-п.н., и, следовательно, $v$ тождественно равна $V(0)+\gamma\left(\mathbf{Q}^{*}\right)=U(\infty)+\gamma\left(\mathbf{Q}^{*}\right)$. В силу (2.14) функция $u$ тождественно равна той же константе. Таким образом, для любой $\mathbf{Q} \in \arg \min \gamma$ выполнено равенство

$$
\sup _{\xi \in \mathscr{A}}\left[\mathbf{E}_{\mathbf{Q}} U(\xi)+\gamma(\mathbf{Q})\right]=U(\infty)+\gamma(\mathbf{Q}),
$$

и мы приходим к противоречию.

Наконец докажем (ix). Предположение на $U$ означает в точности, что $V$ строго выпукла. Пусть $v(y)<+\infty$ и минимум в $(2.12)$ достигается на парах $\left(\eta_{1}, \mathbf{Q}_{1}\right)$ и $\left(\eta_{2}, \mathbf{Q}_{2}\right)$; положим $\eta:=\left(\eta_{1}+\eta_{2}\right) / 2$ и $\mathbf{Q}:=\left(\mathbf{Q}_{1}+\mathbf{Q}_{2}\right) / 2$. Тогда, с одной стороны, в силу выпуклости множеств $\mathscr{D}(y)$ и $\mathscr{Q}$ и имеем

$$
v(y) \leqslant \mathbf{E}_{\mathbf{Q}} V\left(\frac{\eta}{d \mathbf{Q} / d \mathbf{P}}\right)+\gamma(\mathbf{Q}) .
$$

С другой стороны, в силу выпуклости $V$-дивергенции и $\gamma$ находим, что

$$
\begin{aligned}
\mathbf{E}_{\mathbf{Q}} V & \left(\frac{\eta}{d \mathbf{Q} / d \mathbf{P}}\right)+\gamma(\mathbf{Q})=\mathscr{J}_{V}(\eta \cdot \mathbf{P}, \mathbf{Q})+\gamma(\mathbf{Q}) \\
& \leqslant \frac{1}{2}\left[\mathscr{J}_{V}\left(\eta_{1} \cdot \mathbf{P}, \mathbf{Q}_{1}\right)+\mathscr{J}_{V}\left(\eta_{2} \cdot \mathbf{P}, \mathbf{Q}_{2}\right)+\gamma\left(\mathbf{Q}_{1}\right)+\gamma\left(\mathbf{Q}_{2}\right)\right] \\
& =\frac{1}{2}\left[\mathbf{E}_{\mathbf{Q}_{1}} V\left(\frac{\eta_{1}}{d \mathbf{Q}_{1} / d \mathbf{P}}\right)+\gamma\left(\mathbf{Q}_{1}\right)\right]+\frac{1}{2}\left[\mathbf{E}_{\mathbf{Q}_{2}} V\left(\frac{\eta_{2}}{d \mathbf{Q}_{2} / d \mathbf{P}}\right)+\gamma\left(\mathbf{Q}_{2}\right)\right] \\
& =v(y) .
\end{aligned}
$$

Таким образом, неравенства в (3.16) и (3.17) являются равенствами, причем

$$
\begin{gathered}
\gamma(\mathbf{Q})=\frac{\gamma\left(\mathbf{Q}_{1}\right)+\gamma\left(\mathbf{Q}_{2}\right)}{2} \\
\mathbf{E}_{\mathbf{Q}} V\left(\frac{\eta}{d \mathbf{Q} / d \mathbf{P}}\right)=\frac{1}{2}\left[\mathbf{E}_{\mathbf{Q}_{1}} V\left(\frac{\eta_{1}}{d \mathbf{Q}_{1} / d \mathbf{P}}\right)+\mathbf{E}_{\mathbf{Q}_{2}} V\left(\frac{\eta_{2}}{d \mathbf{Q}_{2} / d \mathbf{P}}\right)\right] .
\end{gathered}
$$

Из первого равенства и строгой выпуклости $\gamma$ получаем $\mathbf{Q}=\mathbf{Q}_{1}=\mathbf{Q}_{2}$, после чего второе равенство и строгая выпуклость $V$ влекут $\eta_{1}=\eta_{2}$ Q-п.н.

Дифференцируемость функции $u$ на $(0,+\infty)$ означает, что $v$ строго выпукла на множестве $\left\{y:+\infty>v(y)>\lim _{z \rightarrow+\infty} v(z)\right\}$. Предположим, что это не так и $v$ конечна и линейна на отрезке $\left[y_{1}, y_{2}\right], v\left(y_{2}\right)<v\left(y_{1}\right)$. Пусть минимум в $(2.12)$ достигается на паре $\left(\eta_{1}, \mathbf{Q}_{1}\right)$ при $y=y_{1}$ и на паре $\left(\eta_{2}, \mathbf{Q}_{2}\right)$ при $y=y_{2}$. Аналогично доказательству первого утверждения пункта (ix), см. также доказательство выпуклости функции $v$, получим, что $\mathbf{Q}_{1}=\mathbf{Q}_{2}$ и $\eta_{1}=\eta_{2} \mathbf{Q}_{1}$-п.н., что влечет $v\left(y_{1}\right)=v\left(y_{2}\right)$, т.е. мы пришли к противоречию. 
Утверждение о строгой выпуклости $v$ есть комбинация предыдущего утверждения и пункта (viii).

Теорема 2.2 доказана.

Д ок аз а т ель с тв о п ре дло жени я 2.1. (i) $\Rightarrow$ (ii). Предположим противное. Тогда по теореме Яна [45, теорема 1] существует такая вероятностная мера $\mathbf{Q}^{\prime} \sim \mathbf{Q}$, что $\sup _{\xi \in \mathscr{A}_{+}} \mathbf{E}_{\mathbf{Q}^{\prime}} \xi<\infty$. Поскольку плотность $d \mathbf{Q} / d \mathbf{Q}^{\prime}$ интегрируема по $\mathbf{Q}^{\prime}$, найдется такая возрастающая выпуклая функция $\Phi: \mathbf{R}_{+} \rightarrow \mathbf{R}_{+}$, что $\Phi(0)=0, \lim _{y \rightarrow+\infty} \Phi(y) / y=+\infty$ и $\mathbf{E}_{\mathbf{Q}^{\prime}} \Phi\left(d \mathbf{Q} / d \mathbf{Q}^{\prime}\right)<\infty$. Определим теперь функции $V(y):=y \Phi(1 / y), y>0$, и $U(x):=\inf _{y>0}[V(y)+x y], x \geqslant 0$; тогда $V$ выпукла, $\lim _{y \rightarrow+\infty} V(y) / y=0$, поэтому $U$ удовлетворяет предположению 2.1 и имеет место (2.1). Наконец, $\mathscr{J}_{V}\left(\mathbf{Q}^{\prime}, \mathbf{Q}\right)=\mathbf{E}_{\mathbf{Q}} V\left(d \mathbf{Q}^{\prime} / d \mathbf{Q}\right)=\mathbf{E}_{\mathbf{Q}^{\prime}} \Phi\left(d \mathbf{Q} / d \mathbf{Q}^{\prime}\right)<\infty$, поэтому правая часть (3.5) (с $\left.\gamma=\delta_{\{\mathbf{Q}\}}\right)$ конечна, и из леммы 3.2 мы получаем, что $\sup _{\xi \in \mathscr{A}_{+}} \mathbf{E}_{\mathbf{Q}} U(\xi)<\infty$, при этом $U(\infty)=\lim _{y \downarrow 0} V(y)=+\infty$. Противоречие.

(ii) $\Rightarrow$ (i). Пусть функция полезности $U$ не ограничена сверху. Тогда $V(0)=+\infty$ и для конечности $\mathscr{J}_{V}(\mu, \mathbf{Q}), \mu \in \mathfrak{c a}_{+}$, необходимо, чтобы $\mathbf{Q} \ll \mu$.

С другой стороны, для такой $\mu$ имеем $\mathbf{Q}(d \mathbf{Q} / d \mu>0)=1$, поэтому из (ii) следует, что семейство $\left\{\xi\left(\frac{d \mathbf{Q}}{d \mu}\right)^{-1}: \xi \in \mathscr{A}_{+}\right\}$неограничено по $\mathbf{Q}-$ вероятности. Поэтому

$$
\begin{aligned}
\sup _{\xi \in \mathscr{C}_{+}} \mu(\xi) & =\sup _{\xi \in \mathscr{A}_{+}} \mu(\xi) \geqslant \sup _{\xi \in \mathscr{A}_{+}} \mathbf{E}_{\mathbf{Q}} \frac{\xi}{d \mathbf{Q} / d \mu} \\
& \geqslant \sup _{N} \sup _{\xi \in \mathscr{A}_{+}} N \mathbf{Q}\left(\frac{\xi}{d \mathbf{Q} / d \mu}>N\right)=+\infty,
\end{aligned}
$$

и утверждение вытекает из леммы 3.2 .

Док аз а те ль с тв о п ре д ло же н и я 2.2. Утверждение (i) $\Rightarrow$ (ii) очевидно, так как $U(\lambda x), x \geqslant 0$, - также функция полезности.

(ii) $\Rightarrow$ (iii). Мы воспользуемся теоремой 2.2 с $\gamma=\delta_{\{\mathbf{Q}\}}$. Так как задача максимизации полезности (2.11) с произвольным $x>0$ эквивалентна задаче максимизации полезности в исходной модели $($ с $x=1)$ с функцией полезности $U_{x}(\cdot)=U(x \cdot)$, имеем $u(x)=U(\infty)$ для всех $x>0$, значит, $v(y)=U(\infty)=V(0)$ для всех $y \geqslant 0$. Но $V(y)<V(0)$ для любого $y>0$, поэтому из (2.12) следует, что для всякой $\eta \in \mathscr{D}$ выполнено равенство $\eta=0 \mathbf{Q}$-п.н., т.е. $\eta \mathbf{1}_{\{d \mathbf{Q} / d \mathbf{P}>0\}}=0 \mathbf{P}$-п.н., и (iіi) следует из (2.7).

(iii) $\Rightarrow$ (i). Пусть $\mu \in \mathfrak{c a}_{+}$и $\sup _{\xi \in \mathscr{C}_{+}} \mu(\xi)<\infty$. Так как по условию для любого натурального $N$ найдется такая последовательность $\eta_{n} \in \mathscr{C}_{+}$ что $\lim _{n} \eta_{n} \geqslant N \mathbf{1}_{\{d \mathbf{Q} / d \mathbf{P}>0\}}$, из леммы Фату получаем $\mu(d \mathbf{Q} / d \mathbf{P}>0)=0$, т.е. $\mu$ и $\mathbf{Q}$ сингулярны, откуда $\mathscr{J}_{V}(\mu, \mathbf{Q})=V(0)$. Утверждение вытекает из леммы 3.2 . 


\section{4. Приложения.}

4.1. Приложение А: Некоторые факты из выпуклого анализа. Приведенные ниже результаты можно найти в монографии [18].

Пусть $X$ - хаусдорфово локально выпуклое топологическое векторное пространство над полем действительных чисел, а $X^{\prime}-$ сопряженное к нему пространство. Результат действия функционала $x^{\prime} \in X^{\prime}$ на $x \in X$ будем записывать как $\left\langle x, x^{\prime}\right\rangle$. Преобразованием Фенхеля функции $f: X \rightarrow \mathbf{R} \cup\{+\infty\}$ (или сопряженной к $f$ ) называется функция $f^{*}$ на пространстве $X^{\prime}$, задаваемая равенством

$$
f^{*}\left(x^{\prime}\right)=\sup _{x \in X}\left[\left\langle x, x^{\prime}\right\rangle-f(x)\right] .
$$

Сопряженная функция полунепрерывна снизу в топологии $\sigma\left(X^{\prime}, X\right)$ и выпукла. Теорема Фенхеля-Моро утверждает, что

$$
f(x)=\sup _{x^{\prime} \in X^{\prime}}\left[\left\langle x, x^{\prime}\right\rangle-f^{*}\left(x^{\prime}\right)\right], \quad x \in X,
$$

тогда и только тогда, когда $f$ - полунепрерывная снизу выпуклая функция.

В доказательстве ключевой леммы 3.1 мы использовали теорему двойственности Фенхеля-Рокафеллара (см. [31], [18, § 3.4, теорема 1]), которая утверждает, что если $f, g: X \rightarrow \mathbf{R} \cup\{+\infty\}$ - выпуклые собственные функции и найдется точка $x \in \operatorname{dom} f \cap \operatorname{dom} g$, в которой одна из них непрерывна, то

$$
-\inf _{x \in X}[f(x)+g(x)]=\min _{x^{\prime} \in X^{\prime}}\left[f^{*}\left(-x^{\prime}\right)+g^{*}\left(x^{\prime}\right)\right] .
$$

Другие варианты условий, при которых справедлив этот результат, можно найти в [47, теорема 2.8.7].

4.2. Приложение В: $f$-дивергенция и ее свойства. Пусть $f: \mathbf{R} \rightarrow \mathbf{R} \cup\{+\infty\}-$ собственная полунепрерывная снизу выпуклая функция с $\operatorname{dom} f \subseteq \mathbf{R}_{+}$. В [15] было дано определение $f$-дивергенции $\mathscr{J}_{f}(\mu, \nu)$ конечно-аддитивных функций множества $\mu$ и $\nu$ (заданных на $(\Omega, \mathscr{F}))$, не обязательно удовлетворяющих условию (1.2). Легко видеть, что для $\mu, \nu \in \mathfrak{b a}$ это определение эквивалентно следующему:

$$
\mathscr{J}_{f}(\mu, \nu)=\sup _{\xi, \eta \in L^{\infty}: \eta+f^{*}(\xi) \leqslant 0}[\mu(\xi)+\nu(\eta)],
$$

где $f^{*}$ есть преобразование Фенхеля функции $f$. Из определения следует, что функция $\mathscr{J}_{f}(\mu, \nu)$ на $\mathfrak{b} \mathfrak{a} \times \mathfrak{b} \mathfrak{a}$ принимает значения в $\mathbf{R} \cup\{+\infty\}$, выпукла и полунепрерывна снизу в топологии $\sigma\left(\mathfrak{b} \mathfrak{a} \times \mathfrak{b a}, L^{\infty} \times L^{\infty}\right)$. Другие перечисленные ниже и используемые в данной статье свойства доказаны 
в $[15$, теорема 1$]$. В части утверждений мы ограничимся лишь некоторыми частными случаями, в частности, будет предполагаться, что либо

$$
\lim _{y \uparrow+\infty} \frac{f(y)}{y}=0,
$$

либо

$$
\lim _{y \uparrow+\infty} \frac{f(y)}{y}=+\infty .
$$

Заметим, что если хотя бы одна из мер $\mu$ и $\nu$ не лежит в $\mathfrak{b} \mathfrak{a}_{+}$, то $\mathscr{J}_{f}(\mu, \nu)=+\infty$. Всюду ниже $\mu, \nu \in \mathfrak{b a}_{+}$, a $\mathbf{Q} \in \mathfrak{P}$.

Имеют место следующие соотношения:

$$
\mathscr{J}_{f}(\mu, \nu)=\mathscr{J}_{f}\left(\mu^{\mathrm{r}}, \nu^{\mathrm{r}}\right)+\mathscr{J}_{f}\left(\mu^{\mathrm{s}}, \nu^{\mathrm{s}}\right)
$$

$\mathscr{J}_{f}(\mu, 0)=0$ в случае $(4.2)$ и $\mathscr{J}_{f}(\mu, 0)=+\infty$ в случае $(4.3)$, если $\mu \neq 0$.

Для $\mu, \nu \in \mathfrak{c a}_{+}$

$$
\mathscr{J}_{f}(\mu, \nu)=\int f\left(\frac{d \mu}{d \nu}\right) d \nu
$$

где правая часть интерпретируется по-разному в случаях (4.2) и (4.3). Если $f$ удовлетворяет (4.3), то (4.4) имеет место при $\mu \ll \nu$, а в противном случае $\mathscr{J}_{f}(\mu, \nu)=+\infty$. Если же $f$ удовлетворяет $(4.2)$, то под $d \mu / d \nu$ здесь и далее понимается производная Радона-Никодима $d \mu^{\mathrm{c}} / d \nu$ абсолютно непрерывной компоненты $\mu^{\mathrm{c}}$ из разложения Лебега $\mu$ относительно $\nu$. Заметим, что $\nu(d \nu / d \mathbf{P}=0)=0$ и

$$
\frac{d \mu^{\mathrm{c}}}{d \nu}=\frac{d \mu / d \mathbf{P}}{d \nu / d \mathbf{P}} \quad \nu \text {-п.в. }
$$

Таким образом, для $f$, конечной на $(0,+\infty)$, приведенное определение $f$-дивергенции для счетно-аддитивных мер сводится к классическому определению $f$-дивергенции, введенному И. Чисаром [7].

В частности, из приведенных свойств следует, что

$$
\begin{aligned}
& \mathscr{J}_{f}(\mu, \mathbf{Q})=\infty, \quad \text { если выполнено }(4.3) \text { и } \mu \in \mathfrak{b a}_{+} \backslash \mathfrak{c a}_{+}, \\
& \mathscr{J}_{f}(\mu, \mathbf{Q})=\mathbf{E}_{\mathbf{Q}} f\left(\frac{d \mu^{\mathrm{r}}}{d \mathbf{Q}}\right), \quad \text { если выполнено }(4.2) .
\end{aligned}
$$

В качестве функции $f$ можно взять функцию $V$, которая у нас задается соотношениями (2.1) (когда $U$ конечна на полупрямой) или $(2.20)$ (когда $U$ конечна всюду). Тогда неравенство $\eta+f^{*}(\xi) \leqslant 0$ в (4.1) преобразуется в $\eta \leqslant U(-\xi)$ (где в первом случае $U$ предполагается непрерывной справа в нуле), ср. с определением $\varphi$ в доказательстве леммы 3.1. Двум принципиально различным случаям для функции полезности в терминах функции $V$ отвечают условия (2.3) и (2.21), ср. с (4.2) и (4.3). Более подробно о связи свойств функций $U$ и $V$, а также $u$ и $v$, см. [15, разд. 4]. 


\section{СПИСОК ЛИТЕРАТУРЫ}

1. Bellini F., Frittelli M. On the existence of minimax martingale measures. - Math. Finance, 2002, v. 12, № 1, p. 1-21.

2. Biagini S., Frittelli M. A unified framework for utility maximization problems: an Orlicz space approach. - Ann. Appl. Probab., 2008, v. 18, № 3, p. 929-966.

3. Bismut J.-M. Conjugate convex functions in optimal stochastic control. - J. Math. Anal. Appl., 1973, v. 44, № 2, p. 384-404.

4. Bouchard B., Touzi N., Zeghal A. Dual formulation of the utility maximization problem: the case of nonsmooth utility. - Ann. Appl. Probab., 2004, v. 14, № 2, p. 678717.

5. Brannath $W$., Schachermayer $W$. A bipolar theorem for $L_{+}^{0}(\Omega, \mathscr{F}, \mathbf{P})$. - Lecture Notes in Math., 1999, v. 1709, p. 349-354.

6. Burgert C., Rüschendorf L. Optimal consumption strategies under model uncertainty. - Statist. Decisions, 2005, v. 23, № 1, p. 1-14.

7. Csiszár I. Eine informationstheoretische Ungleichung und ihre Anwendung auf den Beweis der Ergodizität von Markoffschen Ketten. - Magyar Tud. Akad. Mat. Kutató Int. Közl., 1963, v. 8, p. 85-108.

8. Cvitanić J., Schachermayer W., Wang H. Utility maximization in incomplete markets with random endowment. - Finance Stoch., 2001, v. 5, № 2, p. 259-272.

9. Deelstra G., Pham H., Touzi N. Dual formulation of the utility maximization problem under transaction costs. - Ann. Appl. Probab., 2001, v. 11, № 4, p. 1353-1383.

10. Drapeau S., Kupper $M$. Risk preferences and their robust representation. Preprint. Berlin: Humboldt University, 2010.

11. Föllmer H., Gundel A. Robust projections in the class of martingale measures. Illinois J. Math., 2006, v. 50, № 2, p. 439-472.

12. Gilboa I., Schmeidler D. Maxmin expected utility with nonunique prior. - J. Math. Econom., 1989, v. 18, № 2, p. 141-153.

13. Goll T., Rüschendorf L. Minimax and minimal distance martingale measures and their relationship to portfolio optimization. - Finance Stoch., 2001, v. 5, № 4, p. 557581.

14. Gundel A. Robust utility maximization for complete and incomplete market models. - Finance Stoch., 2005, v. 9, № 2, p. 151-176.

15. Гущин A. A. О расширении понятия $f$-дивергенции. - Теория вероятн. и ее примен., 2007, т. 52, в. 3, с. 468-489.

16. Hernández-Hernández D., Schied A. Robust utility maximization in a stochastic factor model. — Statist. Decisions, 2006, v. 24, № 1, p. 109-125.

17. Hernández-Hernández D., Schied A. A control approach to robust utility maximization with logarithmic utility and time-consistent penalties. - Stochastic Process. Appl., 2007, v. 117, № 8, p. 980-1000.

18. Иоффе А. Д., Тихомиров В. М. Теория экстремальных задач. М.: Наука, 1974, $479 \mathrm{c}$.

19. Jouini E., Schachermayer W., Touzi $N$. Law invariant risk measures have the Fatou property. - Advances in Mathematical Economics, v. 9. Tokyo: Springer, 2006, p. 4971.

20. Karatzas I., Kardaras C. The numéraire portfolio in semimartingale financial models. - Finance Stoch., 2007, v. 11, № 4, p. 447-493.

21. Karatzas I., Žitković G. Optimal consumption from investment and random endowment in incomplete semimartingale markets. - Ann. Probab., 2003, v. 31, № 4, p. $1821-1858$.

22. Kramkov D., Schachermayer $W$. The asymptotic elasticity of utility functions and optimal investment in incomplete markets. - Ann. Appl. Probab., 1999, v. 9, № 3, p. $904-950$.

23. Kramkov D., Schachermayer $W$. Necessary and sufficient conditions in the problem of optimal investment in incomplete markets. - Ann. Appl. Probab., 2003, v. 13, № 4, p. 1504-1516.

24. Maccheroni F., Marinacci M., Rustichini A. Ambiguity aversion, robustness, and the variational representation of preferences. - Econometrica, 2006, v. 74, № 6, p. 14471498. 
25. Merton R. C. Lifetime portfolio selection under uncertainty: The continuous-time case. - Rev. Econom. Statist., 1969, v. 51, № 3, p. 247-257.

26. Merton R. C. Optimum consumption and portfolio rules in a continuous-time model. - J. Econom. Theory, 1971, v. 3, № 4, p. 373-413.

27. Морозов И. С. Расширение класса допустимых стратегий в задаче максимизации робастной полезности с конечной на $\mathbf{R}$ функцией полезности. - Обозрение прикл. и промышл. матем., 2010, т. 17, № 5 .

28. Owen M. P., Žitković G. Optimal investment with an unbounded random endowment and utility-based pricing. - Math. Finance, 2009, v. 19, № 1, p. 129-159.

29. Pliska $S$. R. A stochastic calculus model of continuous trading: optimal portfolios. Math. Oper. Res., 1986, v. 11, № 2, p. 370-382.

30. Quenez M.-C. Optimal portfolio in a multiple-priors model. - Seminar on Stochastic Analysis, Random Fields and Applications IV. Basel: Birkhäuser, 2004, p. 291-321. (Progr. Probab., v. 58.)

31. Rockafellar R. T. Extension of Fenchel's duality theorem for convex functions. Duke Math. J., 1966, v. 33, № 1, p. 81-89.

32. Samuelson P.A. Lifetime portfolio selection by dynamic stochastic programming. Rev. Econom. Statist., 1969, v. 51, № 3, p. 239-246.

33. Schachermayer $W$. Optimal investment in incomplete markets when wealth may become negative. - Ann. Appl. Probab., 2001, v. 11, № 3, p. 694-734.

34. Schachermayer $W$. Optimal investment in incomplete financial markets. - Mathematical Finance-Bachelier Congress, 2000 (Paris). Berlin: Springer, 2002, p. 427462.

35. Schied A. On the Neyman-Pearson problem for law-invariant risk measures and robust utility functionals. - Ann. Appl. Probab., 2004, v. 14, № 3, p. 1398-1423.

36. Schied A. Optimal investments for robust utility functionals in complete market models. - Math. Oper. Res., 2005, v. 30, № 3, p. 750-764.

37. Schied A. Risk measures and robust optimization problems. - Stoch. Models, 2006, v. 22 , № 4 , p. $753-831$.

38. Schied A. Optimal investments for risk- and ambiguity-averse preferences: a duality approach. - Finance Stoch., 2007, v. 11, № 1, p. 107-129.

39. Schied A. Robust optimal control for a consumption-investment problem. - Math. Methods Oper. Res., 2008, v. 67, № 1, p. 1-20.

40. Schied A., Föllmer H., Weber S. Robust preferences and robust portfolio choice. Handbook of Numerical Analysis, v. XV. Special Volume: Mathematical Modeling and Numerical Methods in Finance. Amsterdam: Elsevier/North-Holland, 2009, p. $29-87$.

41. Schied A., $W u$ C.-T. Duality theory for optimal investments under model uncertainty. - Statist. Decisions, 2005, v. 23, № 3, p. 199-217.

42. Talay D., Zheng Z. Worst case model risk management. — Finance Stoch., 2002, v. 6, № 4 , p. 517-537.

43. Нейман Дж. фон, Моргенштерн О. Теория игр и экономическое поведение. М.: Наука, 1970, 707 c.

44. Wittmüss $W$. Robust optimization of consumption with random endowment. Stochastics, 2008 , v. 80, № 5, p. 459-475.

45. Yan J. A. Caractérisation d'une classe d'ensembles convexes de $L^{1}$ ou $H^{1}$. - Lecture Notes in Math., 1980, v. 784, p. 220-222.

46. Yan J. A. A numeraire-free and original probability based framework for financial markets. - Proceedings of the International Congress of Mathematicians (Beijing, 2002), v. III. Beijing: Higher Ed. Press, 2002, p. 861-871.

47. Zălinescu C. Convex Analysis in General Vector Spaces. River Edge: World Scientific, 2002, $367 \mathrm{p}$.

48. Žitković G. Utility maximization with a stochastic clock and an unbounded random endowment. - Ann. Appl. Probab., 2005, v. 15, № 1B, p. 748-777.

Поступила в редакцию 16.II. 2010 